Bull. Soc. math. France

133 (4), 2005, p. 459-495

\title{
INÉGALITÉ DE VOJTA GÉNÉRALISÉE
}

\author{
PAR GAËL RÉMOND
}

RÉSUMÉ. - La méthode que Vojta a introduite dans sa preuve de la conjecture de Mordell et que Faltings a étendue pour prouver la conjecture de Lang sur les sous-variétés de variétés abéliennes repose sur une inégalité de hauteurs obtenue par approximation diophantienne. Nous montrons qu'une telle inégalité peut s'énoncer de manière très générale en dehors du contexte des groupes algébriques. Ce faisant, nous lui conférons également plus de souplesse, ce qui conduit à des applications nouvelles même sur les variétés abéliennes.

Abstract (Generalized Vojta inequality). - The method introduced by Vojta to give a different proof of Mordell's conjecture has been generalized by Faltings to establish Lang's conjecture on abelian varieties and then further extended by Vojta to deal with semi-abelian varieties. In each case, the heart of the proof can be summarized in an inequality of heights, obtained via diophantine approximation. Here, we present a generalization of this step. We show it is not necessary to work with algebraic groups for this part and phrase our theorem only in terms of sheaves on projective schemes. This allows us to introduce more parameters in the statement and offers a wider range of applications. In the semi-abelian case, we obtain a variation of Vojta's result which implies Poonen's conjecture. Even in the abelian case, our inequality leads to strengthenings of Faltings' theorem.

Texte reçu le 10 septembre 2003, révisé le 15 octobre 2004, accepté le 18 novembre 2004. GAËL RÉmond, Institut Fourier, UMR 5582, BP 74, 38402 Saint-Martin-d'Hères Cedex (France) • E-mail : Gael.Remond@ujf-grenoble.fr

Classification mathématique par sujets (2000). — 11G35, 11G50, 14G25.

Mots clefs. - Hauteurs, conjectures de Lang. 


\section{Introduction}

1.1. Motivation. - Ce travail s'inscrit dans une lignée amorcée par P. Vojta [16] lorsqu'il a donné en 1990 une nouvelle preuve de la conjecture de Mordell. Nous commençons par réinterpréter sa méthode pour montrer en quoi nous nous proposons de la généraliser.

Soit donc $C$ une courbe projective lisse de genre au moins 2 sur un corps de nombres $K$. Nous considérons la jacobienne $J$ de $C$. Nous supposons pour simplifier que $C$ a un point rationnel de façon à voir $C$ comme un sous-schéma fermé de $J$. Ceci permet d'associer à un entier $a \geq 2$ le morphisme décrit par

$$
\alpha_{a}: C \times C \longrightarrow J, \quad(x, y) \longmapsto a x-y
$$

et il est facile de vérifier que ce morphisme est fini. Le cœur du travail de Vojta peut se voir comme une comparaison entre la hauteur d'un point de $C \times C$ et celle de son image par $\alpha_{a}$. Celle-ci serait banale si nous considérions a comme fixé mais tout l'intérêt ici réside dans l'explicitation de la dépendance en $a$. De manière précise, nous pouvons énoncer, si $h$ est une hauteur de Néron-Tate sur $J$ :

InÉGALITÉ 1. - Il existe des réels $c_{1}, c_{2}, c_{3}>0$ tels que

$$
h(a x-y) \geq c_{1}^{-1}\left(a^{2} h(x)+h(y)\right)
$$

pour tout entier $a \geq c_{2}$ et tout couple $(x, y) \in C(K)^{2}$ avec $h(x) \geq c_{3}$ et $h(y) \geq$ $a^{2} c_{3}$.

Nous nous intéresserons uniquement dans la suite à cette partie de la preuve mais rappelons brièvement comment la finitude de $C(K)$ se déduit d'un tel énoncé. Il s'agit, grâce au théorème de Mordell-Weil, de faire de la géométrie euclidienne dans $J(K) \otimes \mathbb{R}$. En effet, en choisissant $a^{2}$ proche de $h(y) / h(x)$, l'inégalité entraîne que $x$ et $y$ ne peuvent pas être trop proches angulairement. Cela montre qu'il n'y a qu'un nombre fini de points de $C(K)$ de hauteur au moins $c_{3}$ et donc que $C(K)$ lui-même est fini.

L'inégalité ci-dessus constitue donc l'essentiel de la preuve et s'obtient via une construction d'approximation diophantienne assez élaborée. Nous souhaitons généraliser celle-ci et, pour cela, il est commode de travailler en termes de faisceaux inversibles. En effet, dans la situation ci-dessus, si $\mathcal{L}$ est un faisceau ample et symétrique sur $J$ auquel la hauteur $h$ est associée, nous disposons sur $C \times C$ de deux faisceaux $\mathcal{M}_{a}=\alpha_{a}^{*} \mathcal{L}$ et $\mathcal{N}_{a}=p_{1}^{*} \mathcal{L}^{\otimes a^{2}} \otimes p_{2}^{*} \mathcal{L}$, tous deux dépendant de l'entier $a$, et le résultat compare les hauteurs associées à $\mathcal{M}_{a}$ et $\mathcal{N}_{a}$ de manière indépendante de $a:$ nous avons $h_{\mathcal{M}_{a}}(x, y) \geq c_{1}^{-1} h_{\mathcal{N}_{a}}(x, y)$.

Nous percevons ici la première idée motivant notre résultat : la jacobienne $J$ intervient très peu dans cette inégalité. Elle sert certes à définir les faisceaux $\mathcal{M}_{a}$ et $\mathcal{N}_{a}$ mais tout se passe ensuite sur $C \times C$. Cette intuition est confirmée par la preuve et l'on peut donc chercher les hypothèses à mettre

TOME $133-2005-\mathrm{N}^{\mathrm{O}} 4$ 
sur les faisceaux $\mathcal{M}_{a}$ et $\mathcal{N}_{a}$ pour remplacer les données provenant de $J$. Il faut également remarquer que le fait que nous ayons utilisé plus haut une hauteur normalisée n'est pas gênant car il suffit d'ajuster les constantes pour substituer à $h$ une autre hauteur associée à $\mathcal{L}$. Il est par suite possible de formuler l'inégalité seulement en termes de hauteurs de Weil sur $C \times C$.

D'un autre côté, nous allons bien sûr tirer parti des généralisations déjà existantes du travail de Vojta. Tout d'abord, G. Faltings a étendu la méthode au cas des sous-variétés $X$ de dimension quelconque d'une variété abélienne $A$ (voir [5] et [6]) pour obtenir les résultats conjecturés par S. Lang. L'inclusion $X \subset A$ remplace donc $C \subset J$. Ici $X(K)$ n'est pas fini en général mais, si nous introduisons l'ensemble exceptionnel $Z_{X}$ défini comme la réunion des translatés inclus dans $X$ de sous-variétés abéliennes non nulles de $A$, Faltings prouve que $\left(X \backslash Z_{X}\right)(K)$ est fini. Le morphisme

$$
\begin{aligned}
\beta_{a}: X^{m} & \longrightarrow A^{m-1}, \\
x=\left(x_{1}, \ldots, x_{m}\right) & \longmapsto\left(a_{1} x_{1}-a_{2} x_{2}, \ldots, a_{m-1} x_{m-1}-a_{m} x_{m}\right)
\end{aligned}
$$

associé à $a \in(\mathbb{N} \backslash\{0\})^{m}$ où $m=\operatorname{dim} X+1$ généralise $\alpha_{a}$ assez naturellement. Il n'est pas fini mais — dès que la situation n'est pas dégénérée, c'est-à-dire dès que $Z_{X} \neq X$ - il est encore génériquement fini. De la même façon, l'étape principale de la preuve devient (voir [6] ou [9]) :

InÉGALITÉ 2 . - Il existe des réels $c_{1}, c_{2}, c_{3}>0$ tels que

$$
\sum_{i=1}^{m-1} h\left(a_{i} x_{i}-a_{i+1} x_{i+1}\right) \geq c_{1}^{-1} \sum_{i=1}^{m} a_{i}^{2} h\left(x_{i}\right)
$$

pour tous les $a \in(\mathbb{N} \backslash\{0\})^{m}$ et $x \in\left(X \backslash Z_{X}\right)(K)^{m}$ qui vérifient $a_{i} / a_{i+1} \geq c_{2}$ et $a_{i}^{2} h\left(x_{i}\right) \geq a_{1}^{2} c_{3}$.

En termes de faisceaux, l'analogie se poursuit : sur $X^{m}$, nous comparons les hauteurs associées à

$$
\mathcal{M}_{a}=\beta_{a}^{*} \bigotimes_{i=1}^{m-1} p_{i}^{*} \mathcal{L} \quad \text { et } \quad \mathcal{N}_{a}=\bigotimes_{i=1}^{m} p_{i}^{*} \mathcal{L}^{\otimes a_{i}^{2}}
$$

si $\mathcal{L}$ est symétrique et ample sur $A$. Nous pouvons donc envisager une comparaison de hauteurs sur $X^{m}$, pour un schéma projectif $X$ quelconque et un entier $m \geq 2$, entre deux faisceaux vérifiant des conditions ad hoc, toute structure de groupe algébrique ayant disparu. Le travail de Faltings nous montre aussi quelle sera la forme de l'hypothèse principale sur ces faisceaux : il établit en effet que pour tout sous-produit $Y=Y_{1} \times \cdots \times Y_{m}$ de $X^{m}$ qui rencontre $\left(X \backslash Z_{X}\right)^{m}$ il y a une minoration de la forme

$$
\left[\mathcal{M}_{a}\right]^{\operatorname{dim} Y} \cdot Y \geq \theta^{-1}\left[\mathcal{N}_{a}\right]^{\operatorname{dim} Y} \cdot Y
$$

BULLETIN DE LA SOCIÉTÉ MATHÉMATIQUE DE FRANCE 
pour un réel $\theta$ indépendant de $a$ et de $Y$. C'est de cette manière qu'est utilisé le fait que $\beta_{a}$ soit génériquement fini.

Enfin, nous nous inspirons du travail de P. Vojta [17] en 1996 sur les variétés semi-abéliennes. Il a adapté la méthode à ce cas et ceci permit à M. McQuillan de résoudre complètement la conjecture de Lang dans ce cadre. La difficulté principale, à savoir que les schémas en question ne sont pas propres, se résout en travaillant sur un éclatement de $X^{m}$. Nous procédons donc ici de la même façon que dans [12] où nous avons établi une inégalité de Vojta pour les tores.

Nous renvoyons à [17] ou [13] (où le résultat est déduit du théorème démontré ici) pour des détails sur le cas semi-abélien. Écrivons simplement l'inégalité pour illustrer notre propos; il faut décomposer la hauteur en $h=h_{\text {lin }}+h_{\text {quad }}$ (parties linéaire et quadratique correspondant aux comportements torique et abélien, respectivement) et nous avons

$$
\sum_{i=1}^{m-1} h_{\operatorname{lin}}\left(a_{i}^{2} x_{i}-a_{i+1}^{2} x_{i+1}\right)+h_{\mathrm{quad}}\left(a_{i} x_{i}-a_{i+1} x_{i+1}\right) \geq c_{1}^{-1} \sum_{i=1}^{m} a_{i}^{2} h\left(x_{i}\right)
$$

avec des hypothèses analogues à celles du cas abélien (voir [13]).

L'énoncé que nous proposons ci-dessous (voir théorème 1.2) reprend donc les ingrédients que nous venons de présenter. Sur un éclatement de la puissance $X^{m}$ d'un schéma projectif sont introduits deux faisceaux $\mathcal{M}_{a}$ et $\mathcal{N}_{a}$ dépendant d'un élément $a \in(\mathbb{N} \backslash\{0\})^{m}$. Ils vérifient deux hypothèses : celle que nous choisissons de considérer comme secondaire concerne essentiellement la hauteur de sections de $\mathcal{M}_{a}$ et remplace l'existence de formules d'addition dans le cadre des groupes algébriques; la principale est une minoration du nombre d'auto-intersection de $\mathcal{M}_{a}$ sur les éclatés de certains sous-produits de $X^{m}$. La conclusion donne alors une comparaison, indépendante de $a$, entre les hauteurs induites par $\mathcal{M}_{a}$ et $\mathcal{N}_{a}$. Signalons que, plus bas, le faisceau $\mathcal{M}_{a}$ sera en fait noté seulement $\mathcal{M}$ car, au contraire de $\mathcal{N}_{a}$, il ne sera pas entièrement déterminé par a mais pourra être choisi de manière plus souple.

Remarquons que nous avons cité les applications aux points rationnels mais que le théorème 1.2 lui-même concerne tout $\overline{\mathbb{Q}}$ et que les corps de définition des différents objets n'apparaissent jamais. Par ailleurs, l'énoncé améliore légèrement les inégalités 1 et 2 puisque l'hypothèse $a_{i}^{2} h\left(x_{i}\right) \geq a_{1}^{2} c_{3}$ devient seulement $h\left(x_{i}\right) \geq c_{3}$. Enfin, signalons encore que le résultat est effectif, au sens où il fournit des valeurs explicites pour $c_{1}, c_{2}$ et $c_{3}$.

Comme motivation pour un tel résultat général, nous mentionnons deux applications. D'une part, dans le cas semi-abélien, l'inégalité obtenue est employée dans [13] pour montrer la conjecture Mordell-Lang plus Bogomolov de B. Poonen. Rappelons que celle-ci, formulée dans [8], est démontrée dans le cas des variétés abéliennes à la fois par B. Poonen dans ce même article et, indépendamment, par S. Zhang dans [18]. Leur méthode permet également de traiter

TOME $133-2005-\mathrm{N}^{\mathrm{O}} 4$ 
les variétés semi-abéliennes qui sont isogènes au produit d'un tore par une variété abélienne mais ne s'étend pas au cas général. Notre approche dans [13] diffère en ce qu'elle n'utilise pas d'arguments d'équidistribution.

Même dans le cas des variétés abéliennes, la latitude sur $a$ et $\mathcal{M}_{a}$ permet d'aller au-delà de la conjecture de Lang et d'envisager des énoncés plus uniformes. À titre d'exemple et pour rester dans le cadre des points rationnels, le résultat principal de [15], obtenu grâce au théorème 1.2 ci-dessous, entraîne l'énoncé suivant.

Proposition 1.1. - Soient $K$ un corps de nombres, E une courbe elliptique sur $K$ à multiplication complexe et $C$ une courbe projective lisse sur $K$ plongée dans sa jacobienne $J$. Alors, l'ensemble des points $P \in C(\bar{K})$ tels qu'il existe un morphisme surjectif $\varphi: J \rightarrow E^{2}$ avec $\varphi(P)$ rationnel sur $K$ est fini.

Dans ce cas, la souplesse de l'énoncé de l'inégalité permet de l'employer pour minorer une hauteur de la forme $h(\varphi(a x-y))$ sur $C \times C$ et de préciser la dépendance en $\varphi$.

1.2. Notations. - Nous définissons la hauteur de Weil (logarithmique et absolue) d'une partie finie $F \subset \overline{\mathbb{Q}}$ comme

$$
h(F)=\sum_{v} \frac{\left[K_{v}: \mathbb{Q}_{v}\right]}{[K: \mathbb{Q}]} \log |F|_{v}
$$

où $K$ désigne le corps de nombres $\mathbb{Q}(F)$ et $|F|_{v}=\max _{x \in F}|x|_{v}$ pour toute place $v$ de $K$. Nous étendons les notations $|P|_{v}$ et $h(P)$ à un polynôme $P$ en l'identifiant à la famille de ses coefficients. De même la hauteur $h(y)$ d'un point fermé $y$ de $\mathbb{P}_{\overline{\mathbb{Q}}}^{n}$ est celle d'un système quelconque de coordonnées de $y$.

Cette hauteur des points est la notion cruciale dans l'énoncé ci-après mais nous utiliserons aussi celle de hauteur projective d'un sous-schéma fermé $X \hookrightarrow \mathbb{P} \frac{n}{\mathbb{Q}}$ notée $h(X)$ (voir [3] ou [10]). Il faut signaler que, pour un point fermé $x$, la hauteur $h(\{x\})$ ainsi introduite diffère de la précédente $h(x)$ (elle correspond à un choix de normes euclidiennes à l'infini) mais nous avons toujours $h(x) \leq h(\{x\})$.

Enfin, si $a \in \mathbb{N}^{m}$ pour un entier $m \geq 1$, nous notons systématiquement $a=\left(a_{1}, \ldots, a_{m}\right)$ et utilisons également $|a|=a_{1}+\cdots+a_{m}$.

1.3. Énoncé principal. — Soient $X$ un schéma projectif sur $\overline{\mathbb{Q}}$, intègre et de dimension non nulle, $\iota: X \hookrightarrow \mathbb{P}_{\overline{\mathbb{Q}}}^{N}$ une immersion fermée et $\mathcal{L}=\iota^{*} \mathcal{O}(1)$. Nous utiliserons le degré de $X$ dans ce plongement (égal au nombre d'intersection $\left.[\mathcal{L}]^{\operatorname{dim} X} \cdot X\right)$ et sa hauteur $h(X)$. 
Soit $\omega \in \mathbb{Z}$ avec $\omega \geq-1$ et notons $\omega^{\prime}=3+\omega$. Soit ensuite $m \geq 2$ un entier et posons pour un entier $u \leq m \operatorname{dim} X$

$$
\psi(u)=\prod_{j=u+1}^{m \operatorname{dim} X}\left(\omega^{\prime} j+1\right) .
$$

Si $t_{1}, t_{2}, M$ et $\theta$ sont des entiers et $\delta$ un réel, tous au moins égaux à 1 , nous posons

$$
\Lambda=\theta\left(2 t_{1} m \operatorname{dim} X\right)^{m \operatorname{dim} X}(N+1)(\operatorname{deg} X)^{\omega^{\prime} m}
$$

puis

$$
\begin{aligned}
& c_{1}=4 \Lambda^{(1+\omega) \psi(1)-\omega}, \\
& c_{2}=\Lambda^{\psi(0)} \quad \text { et } \\
& c_{3}=\left(M t_{2}\right)^{m \operatorname{dim} X} \Lambda^{2 \psi(0)} \max (h(X), \delta) .
\end{aligned}
$$

Au-delà des valeurs précises de ces constantes, il importe surtout qu'elles soient indépendantes des données que nous introduisons maintenant.

Nous nous intéressons à un triplet $(a, \mathcal{X}, \mathcal{M})$ où $a \in(\mathbb{N} \backslash\{0\})^{m}, \mathcal{X}$ est un schéma intègre et projectif sur $\overline{\mathbb{Q}}$ muni d'un morphisme propre et birationnel $\pi: \mathcal{X} \rightarrow X^{m}$ et $\mathcal{M}$ un faisceau inversible sur $\mathcal{X}$. Nous noterons $U$ l'ouvert de $X^{m}$ au-dessus duquel $\pi$ est un isomorphisme et nous nous permettrons de le voir aussi comme ouvert de $\mathcal{X}$. Soit encore $\mathcal{N}_{a}=\pi^{*} \bigotimes_{i=1}^{m} p_{i}^{*} \mathcal{L}^{\otimes a_{i}}$ où $p_{i}: X^{m} \rightarrow X$ est la $i$-ième projection pour $1 \leq i \leq m$.

Nous faisons l'hypothèse qu'il existe un quadruplet $\left(\iota^{\prime}, j_{1}, j_{2}, \Sigma\right)$ où $\iota^{\prime}: \mathcal{X} \hookrightarrow \mathbb{P}_{\overline{\mathbb{Q}}}^{N^{\prime}}$ est une immersion fermée (pour un certain $N^{\prime} \in \mathbb{N}$ ) et, si $\mathcal{P}=\iota^{\prime *} \mathcal{O}(1), j_{1}$ et $j_{2}$ sont respectivement des injections $\mathcal{P} \hookrightarrow \mathcal{N}_{a}^{\otimes t_{1}}$ et $\mathcal{P} \otimes \mathcal{M}^{\otimes-1} \hookrightarrow \mathcal{N}_{a}^{\otimes t_{2}}$ tandis que $\Sigma$ est une famille de sections globales de $\mathcal{P} \otimes \mathcal{M}^{\otimes-1}$ de cardinal $M$ qui engendre ce faisceau sur $\mathcal{X}$. Nous supposons enfin d'une part que les images par $j_{1}$ des coordonnées de $\mathbb{P}_{\overline{\mathbb{Q}}}^{N^{\prime}}$ (vues comme sections de $\mathcal{P}$ ) s'écrivent comme des monômes sans coefficient de multidegré $t_{1} a$ en les coordonnées de $\mathbb{P}_{\overline{\mathbb{Q}}}^{N}$ (vues comme sections des différents $\pi^{*} p_{i}^{*} \mathcal{L}$ ) et d'autre part que les éléments de $j_{2}(\Sigma)$ s'écrivent comme des polynômes $P_{1}, \ldots, P_{M}$ de multidegré $t_{2} a$ en ces mêmes coordonnées de sorte que $h\left(P_{1}, \ldots, P_{M}\right) \leq \delta|a|$.

Si nous notons $U^{\prime}$ l'ouvert (non vide) de $U$ au-dessus duquel $j_{1}$ et $j_{2}$ sont des isomorphismes, $h_{\mathcal{L}}=h \circ \iota: X(\overline{\mathbb{Q}}) \rightarrow \mathbb{R}$ la hauteur induite par $\iota$ et, pour $y \in \mathcal{X}(\overline{\mathbb{Q}}), h_{\mathcal{M}}(y)=h\left(\iota^{\prime}(y)\right)-h(\Sigma(y))$ qui donnent bien des hauteurs de Weil associées à $\mathcal{L}$ et $\mathcal{M}$, nous pouvons énoncer le :

ThÉORÈme 1.2. - Soit $x=\left(x_{1}, \ldots, x_{m}\right) \in U^{\prime}(\overline{\mathbb{Q}})$ un point tel que, pour tout produit $Y=Y_{1} \times \cdots \times Y_{m}$ de sous-schémas fermés intègres de $X$ contenant $x$,

TOME $133-2005-\mathrm{N}^{\mathrm{O}} 4$ 
nous ayons, en notant $\mathcal{Y}$ l'adhérence de $Y \cap U$ dans $\mathcal{X}$, la minoration

$$
[\mathcal{M}]^{\operatorname{dim} Y} \cdot \mathcal{Y} \geq \theta^{-1} \prod_{i=1}^{m}\left(\operatorname{deg} Y_{i}\right)^{-\omega} a_{i}^{\operatorname{dim} Y_{i}}
$$

Alors on a

$$
\sum_{i=1}^{m} a_{i} h_{\mathcal{L}}\left(x_{i}\right) \leq c_{1} h_{\mathcal{M}}(x)
$$

dès que $c_{2} a_{i+1} \leq a_{i}($ pour $1 \leq i<m)$ et $c_{3} \leq h_{\mathcal{L}}\left(x_{i}\right)($ pour $1 \leq i \leq m)$.

1.4. Remarques. - En premier lieu, disons que le paramètre $\omega$ introduit dans le théorème vaut 0 dans les applications que nous avons citées. Toutefois, sa valeur naturelle serait plutôt -1 puisque dans ce cas la minoration du nombre d'intersection s'écrit $[\mathcal{M}]^{\operatorname{dim} Y} \cdot \mathcal{Y} \geq \theta^{-1}\left[\mathcal{N}_{a}\right]^{\operatorname{dim} Y} \cdot \mathcal{Y}$ (qui correspond mieux à l'inégalité de la forme $h_{\mathcal{M}} \geq c_{1}^{-1} h_{\mathcal{N}_{a}}$ qui suit); de plus ce choix a l'avantage de fournir une constante $c_{1}$ plus raisonnable. Et, en effet, comme nous l'avons dit, G. Faltings [6] montre qu'il est possible de choisir $\omega=-1$ dans le cas abélien. Malheureusement, le calcul explicite de $\theta$ ne semble pas aisé dans ce cas-là et l'on risque de reperdre ce que l'on avait gagné sur $c_{1}$. Pour cette raison, le choix fait dans l'effectivisation [9] du travail de Faltings correspond à $\omega=0$ et il se trouve alors que $\theta=1$ convient. D'un autre côté, la possibilité de choisir $\omega \geq 1$ dans le théorème 1.2 garantit une plus grande liberté dans d'autres applications (c'est le cas notamment dans [14] qui vise à généraliser [15] en dimension supérieure).

Les hypothèses un peu imbriquées qui concernent $\mathcal{P}, j_{1}$ et $j_{2}$ sont nécessaires dans le cas semi-abélien (voir [13]). Elles se simplifient lors des applications à un cadre abélien : en effet, il n'est pas besoin d'éclatement et l'on utilise $\pi=\mathrm{id}_{X^{m}}$; de la sorte, choisir $\mathcal{P}=\mathcal{N}_{a}^{\otimes t_{1}}$ devient possible, $j_{1}$ est donc l'identité et avec $t_{1}=t_{2}$ l'application $j_{2}$ correspond seulement à une section globale de $\mathcal{M}$.

La démonstration que nous donnons du théorème 1.2 suit les mêmes idées que celles de [9] et [12]. En particulier, elle s'inspire, outre des travaux déjà cités de Vojta et Faltings, de la réécriture qu'a donnée E. Bombieri du cas des courbes dans [2]. Dans un but de lisibilité, nous n'avons pas souhaité renvoyer au cours de la démonstration le lecteur aux énoncés de [9] et [12]. En effet, bien que ceux du présent texte en soient proches, il est souvent nécessaire de généraliser ou de modifier les arguments et nous les avons donc redonnés précisément.

Le principe reste de construire des sous-schémas $Y=Y_{1} \times \cdots \times Y_{m}$ de hauteur contrôlée et de dimension de plus en plus petite (à partir de $Y=X^{m}$ ). À chaque étape, l'hypothèse sur le nombre d'intersection permet d'assurer qu'un faisceau de la forme $\mathcal{M}^{\otimes d} \otimes \mathcal{N}_{a}^{\otimes-d^{\prime}}$ (avec $d, d^{\prime} \in \mathbb{N}$ ) a suffisamment de sections globales sur $\mathcal{Y}$ (voir premier paragraphe de la partie 4). Grâce au contrôle de la hauteur de $\mathcal{M}$ (à travers $j_{2}$ ), nous en déduisons par un lemme de Siegel l'existence d'une 
section $s \neq 0$ de petite hauteur (deuxième paragraphe de la partie 4 ). Moyennant des estimations locales, nous voyons ensuite que, si $s$ s'annule peu en $x$, la hauteur correspondante est minorée et cela montre l'inégalité de Vojta qui est notre but (voir les deux premiers paragraphes de la partie 5). Sinon $s$ s'annule à un ordre élevé et alors le théorème du produit de Faltings s'applique : par suite, $x$ appartient à un sous-produit $Y^{\prime}=Y_{1}^{\prime} \times \cdots \times Y_{m}^{\prime}$ de hauteur contrôlée auquel on réapplique ce qui précède. À terme, soit le théorème est établi, soit le produit s'est réduit à $\left\{x_{1}\right\} \times \cdots \times\left\{x_{m}\right\}$. Il suffit de faire en sorte que cela n'arrive pas en choisissant $c_{3}$ suffisamment grand pour que l'hypothèse $h_{\mathcal{L}}\left(x_{i}\right) \geq c_{3}$ soit contradictoire avec le contrôle de la hauteur du produit. Notons que, formellement, nous ne raisonnerons pas par récurrence mais considérerons tout de suite le plus petit produit $Y$ satisfaisant les bornes de degré et hauteur de sorte que l'impossibilité de contruire un sous-produit montrera le théorème.

Signalons ici deux notations utilisées dans tout le texte. Nous désignons d'une part par $K(X)$ le corps des fonctions d'un sous-schéma intègre $X$ quelconque. D'autre part, si $W_{0}, \ldots, W_{n}$ sont les coordonnées de $\mathbb{P}_{\overline{\mathbb{Q}}}^{n}$ et $I$ un idéal homogène de $\overline{\mathbb{Q}}\left[W_{0}, \ldots, W_{n}\right]$, nous noterons $V(I)$ le fermé de $\mathbb{P} \frac{n}{\mathbb{Q}}$ correspondant; de plus, si $F$ est un élément homogène de cet anneau, nous posons $D_{+}(F)=\mathbb{P}_{\overline{\mathbb{Q}}}^{n} \backslash V(F)$.

\section{Préliminaires}

Cette partie rassemble plusieurs résultats auxiliaires intervenant dans la démonstration du théorème 1.2 mais qui peuvent s'énoncer en dehors de celle-ci. Nous nous attachons dans un premier paragraphe à décrire certains éléments de l'idéal d'un sous-schéma fermé de $\mathbb{P}_{\overline{\mathbb{Q}}}^{n}$ : il s'agit essentiellement d'une normalisation de Nœether contrôlée (disons au sens de la hauteur) et nos résultats sont voisins de [4, chap. 2]. Par commodité, nous présentons ce procédé comme un changement de plongement, via un automorphisme de $\mathbb{P} \frac{n}{\mathbb{Q}}$.

Nous donnons ensuite deux lemmes qui permettront de manipuler les polynômes obtenus. Le premier s'intéresse à la hauteur du passage au quotient par de tels polynômes et le second à une majoration locale des dérivées d'un élément de ce quotient.

2.1. Plongements adaptés. - Dans un premier temps, nous notons $V_{0}$, $V_{1}, \ldots, V_{n}$ les coordonnées de $\mathbb{P}_{\bar{Q}}^{n}$.

Lorsque $Z$ est un schéma intègre, projectif sur $\overline{\mathbb{Q}}$, de dimension $u<n$, nous dirons ici qu'une immersion fermée $Z \hookrightarrow \mathbb{P}_{\overline{\mathbb{Q}}}^{n}$ est adaptée à $Z$ si

1) $Z \cap V\left(V_{0}, \ldots, V_{u}\right)=\varnothing$ et

2) $K(Z)$ est engendré par les images de $V_{1} / V_{0}, \ldots, V_{u+1} / V_{0}$.

Remarquons que la première condition donne naissance à un morphisme fini $\rho: Z \rightarrow \mathbb{P}_{\overline{\mathbb{Q}}}^{u}$ en composant l'immersion fermée $Z \hookrightarrow \mathbb{P}_{\overline{\mathbb{Q}}}^{n} \backslash V\left(V_{0}, \ldots, V_{u}\right)$ avec

TOME $133-2005-\mathrm{N}^{\mathrm{O}} 4$ 
la projection linéaire $\mathbb{P}_{\overline{\mathbb{Q}}}^{n} \backslash V\left(V_{0}, \ldots, V_{u}\right) \rightarrow \mathbb{P}_{\overline{\mathbb{Q}}}^{u}$ (cette dernière étant un morphisme affine, il en est de même de $\rho$ qui est propre donc fini). En particulier $\rho$ est surjectif (par dimension) donc $Z \cap D_{+}\left(V_{0}\right) \neq \varnothing$, ce qui donne

$$
\Gamma\left(D_{+}\left(V_{0}\right), \mathcal{O}_{\mathbb{P}} \frac{n}{\mathbb{Q}}\right)=\overline{\mathbb{Q}}\left[V_{1} / V_{0}, \ldots, V_{n} / V_{0}\right] \longrightarrow \Gamma\left(D_{+}\left(V_{0}\right) \cap Z, \mathcal{O}_{Z}\right) \longleftrightarrow K(Z) .
$$

Nous pouvons ainsi parler de l'image $v_{i}$ de $V_{i} / V_{0}$ dans $K(Z)$. On a toujours $K(Z)=\overline{\mathbb{Q}}\left(v_{1}, \ldots, v_{n}\right)$. Le fait que $\rho$ soit fini et surjectif montre que $v_{1}, \ldots, v_{u}$ sont algébriquement indépendants et que chaque $v_{i}$ est entier sur $\overline{\mathbb{Q}}\left[v_{1}, \ldots, v_{u}\right]$. Le degré de $\rho$ vaut $\left[K(Z): \overline{\mathbb{Q}}\left(v_{1}, \ldots, v_{u}\right)\right]$ et coïncide avec le degré projectif de $Z \hookrightarrow \mathbb{P} \frac{n}{\mathbb{Q}}$. Le lemme ci-après montre que, sous l'hypothèse 1 ), nous pouvons facilement écrire des relations de dépendance intégrale pour toute combinaison linéaire des $v_{i}$.

Rappelons que, si $Z \hookrightarrow \mathbb{P}_{\overline{\mathbb{Q}}}^{n}$ est de degré $D$, toute forme éliminante $f$ de $Z$ est un polynôme multihomogène de multidegré $(D, \ldots, D)$ en $u+1$ groupes de $n+1$ variables. Chacun de ces groupes correspond naturellement à une forme linéaire et, si $L_{0}, \ldots, L_{u}$ sont de telles formes, $f\left(L_{0}, \ldots, L_{u}\right)$ désigne l'image par $f$ de la famille de leurs coefficients.

Lemme 2.1. - Soit $Z$ un sous-schéma fermé intègre de $\mathbb{P}_{K}^{n}$. On note $u=$ $\operatorname{dim} Z, D=\operatorname{deg} Z$ et $f$ une forme éliminante de $Z$ telle que $f\left(V_{0}, \ldots, V_{u}\right)=1$. Pour $\mu \in \overline{\mathbb{Q}}^{n+1}$, on définit $P_{\mu}\left(X_{0}, \ldots, X_{u+1}\right)$ comme

$$
X_{0}^{-D u} f\left(X_{u+1} V_{0}-\sum_{i=0}^{n} \mu_{i} X_{0} V_{i}, X_{0} V_{1}-X_{1} V_{0}, \ldots, X_{0} V_{u}-X_{u} V_{0}\right) .
$$

Alors $P_{\mu}$ est un polynôme homogène de degré $D$ dans lequel le coefficient de $X_{u+1}^{D}$ est 1 ; il s'écrit comme la puissance d'un polynôme irréductible et le polynôme

$$
P_{\mu}\left(V_{0}, \ldots, V_{u}, \sum_{i=0}^{n} \mu_{i} V_{i}\right)
$$

est élément de l'idéal de $Z$.

Démonstration. - La non-nullité de $f\left(V_{0}, \ldots, V_{u}\right)$ est équivalente à la condition $Z \cap V\left(V_{0}, \ldots, V_{u}\right)=\varnothing$. Écrivons pour cette preuve

$$
W=\sum_{i=0}^{n} \mu_{i} V_{i}
$$

Puisque $Z \cap V\left(V_{0}, \ldots, V_{u}, W\right)=\varnothing$, ces coordonnées définissent un morphisme fini $\pi_{\mu}: Z \rightarrow \mathbb{P}_{\overline{\mathbb{Q}}}^{u+1}$. Ainsi $\pi_{\mu}(Z)$ est une hypersurface irréductible de $\mathbb{P}_{\overline{\mathbb{Q}}}^{u+1}$ et s'écrit donc $V\left(F_{\mu}\right)$ pour un certain polynôme homogène irréductible $F_{\mu}$ de $\overline{\mathbb{Q}}\left[X_{0}, \ldots, X_{u+1}\right]$ (déterminé à la multiplication par un élément de $\overline{\mathbb{Q}}^{\times}$près).

BULlETIN DE LA SOCiÉtÉ MATHÉMATIQUE DE FRANCE 
Comme $Z \cap V\left(V_{0}, \ldots, V_{u}\right)=\varnothing$ entraîne $\pi_{\mu}(Z) \cap V\left(X_{0}, \ldots, X_{u}\right)=\varnothing$ qui se réécrit $F_{\mu}(0, \ldots, 0,1) \neq 0$, nous constatons $\operatorname{deg}_{X_{u+1}} F_{\mu}=\operatorname{deg} F_{\mu}$. Notons

$$
P_{\mu}^{\prime}=f\left(X_{u+1} V_{0}-X_{0} W, X_{0} V_{1}-X_{1} V_{0}, \ldots, X_{0} V_{u}-X_{u} V_{0}\right) .
$$

Si $x_{0}, \ldots, x_{u+1}$ sont des éléments de $\overline{\mathbb{Q}}$ avec $x_{0} \neq 0$, les zéros communs des formes linéaires $x_{u+1} V_{0}-x_{0} W$ et $x_{0} V_{i}-x_{i} V_{0}$ (avec $1 \leq i \leq u$ ) sont les éléments de $\mathbb{P}_{\overline{\mathbb{Q}}}^{n}(\overline{\mathbb{Q}})$ se projetant sur $x=\left(x_{0}: \cdots: x_{u+1}\right) \in \mathbb{P}_{\overline{\mathbb{Q}}}^{u+1}(\overline{\mathbb{Q}})$. Par conséquent (théorème de l'élimination)

$$
\begin{aligned}
P_{\mu}^{\prime}\left(x_{0}, \ldots, x_{u+1}\right)=0 & \Longleftrightarrow \pi_{\mu}^{-1}(x)(\overline{\mathbb{Q}}) \neq \varnothing \\
& \Longleftrightarrow x \in \pi_{\mu}(Z)(\overline{\mathbb{Q}}) \Longleftrightarrow F_{\mu}\left(x_{0}, \ldots, x_{u+1}\right)=0
\end{aligned}
$$

(sous l'hypothèse $x_{0} \neq 0$ ). Ceci montre que l'on peut écrire

$$
P_{\mu}^{\prime}=\lambda X_{0}^{a} F_{\mu}^{b}
$$

avec $\lambda \in \overline{\mathbb{Q}}^{\times}$et $a, b \in \mathbb{N}$. ’̀ présent le coefficient de $X_{0}^{D u} X_{u+1}^{D}$ dans $P_{\mu}^{\prime}$ est facilement, par multihomogénéité,

$$
f\left(V_{0}, V_{1}, \ldots, V_{u}\right)=1 .
$$

Par suite $D=\operatorname{deg}_{X_{u+1}} P_{\mu}^{\prime}=b \operatorname{deg}_{X_{u+1}} F_{\mu}=b \operatorname{deg} F_{\mu}=\operatorname{deg} P_{\mu}^{\prime}-a$ et cela entraîne $a=D u$. Ainsi $P_{\mu}$ est effectivement un polynôme et c'est (à une constante près) une puissance de $F_{\mu}$. Puisque $\pi_{\mu}(Z)=V\left(F_{\mu}\right)$ nous avons

$$
Z \subset \pi_{\mu}^{-1} V\left(F_{\mu}\right)=V\left(F_{\mu}\left(V_{0}, \ldots, V_{u}, W\right)\right)=V\left(P_{\mu}\left(V_{0}, \ldots, V_{u}, W\right)\right),
$$

ce qui termine la preuve.

Nous souhaitons ensuite passer d'un plongement projectif donné à un plongement adapté. Pour cela, associons à une matrice $M \in \mathrm{GL}_{n+1}(\overline{\mathbb{Q}})$ l'automorphisme $\chi: \mathbb{P} \frac{n}{\mathbb{Q}} \rightarrow \mathbb{P}_{\overline{\mathbb{Q}}}^{n}$ qu'elle définit. Si $\varphi: Z \hookrightarrow \mathbb{P}_{\overline{\mathbb{Q}}}^{n}$ est une immersion fermée, nous dirons que $M$ est adaptée à $\varphi$ si l'immersion $\chi \circ \varphi$ est adaptée à $Z$.

Pour clarifier les notations, nous choisissons de désigner par $W_{0}, \ldots, W_{n}$ les coordonnées de $\mathbb{P}_{K}^{n}$ à la source de $\chi$ et $V_{0}, \ldots, V_{n}$ celles de son but. De la sorte, si $m_{i, j}$ sont les coefficients de $M$ et $\widetilde{m}_{i, j}$ ceux de $M^{-1}$, nous avons, via $\chi$, les relations

$$
V_{i}=\sum_{j=0}^{n} m_{i, j} W_{j} \quad \text { et } \quad W_{i}=\sum_{j=0}^{n} \tilde{m}_{i, j} V_{j} .
$$

La proposition ci-dessous montre que l'on peut trouver une matrice adaptée de petite taille. Nous y utilisons les faits élémentaires suivants :

- si $f \in \overline{\mathbb{Q}}\left[X_{1}, \ldots, X_{n}\right] \backslash\{0\}$ et $E \subset \overline{\mathbb{Q}} \operatorname{avec} \operatorname{Card}(E)>\max _{i} \operatorname{deg}_{X_{i}} f$, alors il existe $x \in E^{n}$ tel que $f(x) \neq 0$;

- si $f \in \overline{\mathbb{Q}}\left[X_{0}^{(1)}, \ldots, X_{m_{1}}^{(1)}, \ldots, X_{0}^{(n)}, \ldots, X_{m_{n}}^{(n)}\right] \backslash\{0\}$ est multihomogène et $E \subset \overline{\mathbb{Q}}$ avec $0 \in E$ et $\operatorname{Card}(E) \geq \max \left(2, \max _{i} \operatorname{deg}_{X^{(i)}} f\right)$, alors il existe $x \in E^{m_{1}+\cdots+m_{n}+n}$ tel que $f(x) \neq 0$. 
Proposition 2.2. - Soit $\varphi: Z \rightarrow \mathbb{P}_{\overline{\mathbb{Q}}}^{n}$ un sous-schéma fermé intègre et notons $D=\operatorname{deg} Z$. Il existe une matrice $M \in \mathrm{GL}_{n+1}(\overline{\mathbb{Q}})$ adaptée à $\varphi$ dont les coefficients appartiennent à $E=\left\{a \in \mathbb{Z} ;|a| \leq \frac{1}{2} \max (D, 2)\right\}$.

Démonstration. - Avec les notations qui précèdent, la première condition à établir s'écrit $\chi \circ \varphi(Z) \cap V\left(V_{0}, \ldots, V_{u}\right)=\varnothing$. Si nous notons $f$ une forme éliminante de $Z$ et voyons les $V_{i}$ comme des formes linéaires en les $W_{j}$, ceci se traduit par

$$
f\left(V_{0}, \ldots, V_{u}\right) \neq 0 .
$$

Puisque $f$ est multihomogène de degré $D$ en chaque groupe de variables, nous pouvons choisir les $m_{i, j}$ pour $0 \leq i \leq u$ et $0 \leq j \leq n$ à coefficients dans $E$ de telle sorte que cette condition soit remplie. Les coordonnées $V_{0}, \ldots, V_{u}$ ainsi fixées sont alors automatiquement indépendantes et il existe des indices $k_{u+1}, \ldots, k_{n}$ tels que la famille $V_{0}, \ldots, V_{u}, W_{k_{u+1}}, \ldots, W_{k_{n}}$ soit libre. Choisissons encore $V_{i}=W_{k_{i}}$ si $i>u+1$ (notons que 0 et 1 sont éléments de $E$ ) et

$$
V_{u+1}=W_{k_{u+1}}+\sum_{j=u+2}^{n} m_{u+1, k_{j}} W_{k_{j}} .
$$

Cela assure que la matrice $M$ est inversible et il reste à voir qu'il est possible de trouver les coefficients $m_{u+1, k_{j}}$ dans $E$ de façon que l'image $x$ de $V_{u+1} / V_{0}$ engendre $K(Z)$ au-dessus de $\overline{\mathbb{Q}}\left(\left(V_{i} / V_{0}\right)_{1 \leq i \leq u}\right)$.

Si $\sigma_{1}, \ldots, \sigma_{D}$ sont les différents plongements de cette extension de corps dans une clôture normale, l'élément $x$ l'engendre si et seulement si

$$
\prod_{i=2}^{D}\left(\sigma_{i}(x)-\sigma_{1}(x)\right) \neq 0 .
$$

Le membre de gauche est un polynôme de degré $D-1$ en les coefficients $m_{u+1, k_{j}}$ cherchés et il n'est pas nul en vertu du fait que la famille des différents $W_{k_{j}} / V_{0}$ engendre l'extension considérée. Par suite, il est effectivement possible de choisir ces coefficients dans $E$.

Pour un plongement $\varphi: Z \rightarrow \mathbb{P}_{\overline{\mathbb{Q}}}^{n}$, nous fixons désormais une matrice $M$ adaptée comme dans l'énoncé. Puisque les coefficients de $M$ sont bornés, nous pouvons maintenant contrôler les relations de dépendance du lemme 2.1.

LEMme 2.3. - Avec les hypothèses et les notations qui précèdent, il existe des polynômes homogènes notés $P_{0}, \ldots, P_{n}, Q_{0}, \ldots, Q_{n}$ de $\overline{\mathbb{Q}}\left[X_{0}, \ldots, X_{u+1}\right]$ de degré $D$ dans lesquels le coefficient de $X_{u+1}^{D}$ est 1 , chacun égal à la puissance d'un polynôme irréductible de telle sorte que

1) si $B$ désigne la famille de tous leurs coefficients, on a

$$
h(B) \leq h_{\varphi}(Z)+D(u+1) \log D(n+1) ;
$$

BULletin DE LA SOCiÉtÉ MATHÉmATiQUe DE FRANCE 
2) pour tout indice $j$ avec $0 \leq j \leq n$, les polynômes $P_{j}\left(V_{0}, \ldots, V_{u}, V_{j}\right)$ et $Q_{j}\left(V_{0}, \ldots, V_{u}, W_{j}\right)$ sont éléments de l'idéal de $\chi \circ \varphi(Z)$.

Démonstration. - Soit $f^{\prime}$ l'unique forme éliminante de $\chi(\varphi(Z))$ telle que l'on ait $f^{\prime}\left(V_{0}, \ldots, V_{u}\right)=1$. Posons encore $L_{i}=X_{0} V_{i}-X_{i} V_{0}$ pour $1 \leq i \leq u$. Le lemme 2.1 nous montre que

$$
P=X_{0}^{-D u} f^{\prime}\left(L_{0}, L_{1}, \ldots, L_{u}\right)
$$

fournit $P_{j}$ si $L_{0}=X_{u+1} V_{0}-X_{0} V_{j}$ et $Q_{j}$ si $L_{0}=X_{u+1} V_{0}-\sum_{k=0}^{n} \widetilde{m}_{j, k} X_{0} V_{k}$ satisfaisant toutes les conditions sauf peut-être la majoration de hauteur. Par le théorème de l'élimination, il existe une forme éliminante $f$ de $\varphi(Z)$ telle que, pour toutes formes linéaires $L_{0}, \ldots, L_{u}$,

$$
f^{\prime}\left(L_{0}, \ldots, L_{u}\right)=f\left(L_{0}(M \cdot), \ldots, L_{u}(M \cdot)\right) .
$$

Avec les choix qui précèdent, $L_{i}(M \cdot)=\sum_{k=0}^{n}\left(m_{0, k} X_{i}-m_{i, k} X_{0}\right) W_{k}$ pour $1 \leq i \leq u$; dans le cas de $P_{j}$ la formule pour $L_{0}(M \cdot)$ est analogue tandis que pour $Q_{j}$ nous avons

$$
\begin{aligned}
L_{0}(M \cdot) & =X_{u+1}\left(\sum_{i=0}^{n} m_{0, i} W_{i}\right)-X_{0}\left(\sum_{k=0}^{n} \sum_{i=0}^{n} \widetilde{m}_{j, k} m_{k, i} W_{i}\right) \\
& =X_{u+1}\left(\sum_{i=0}^{n} m_{0, i} W_{i}\right)-X_{0} W_{j}=\sum_{i=0}^{n}\left(m_{0, i} X_{u+1}-\delta_{i, j} X_{0}\right) W_{i} .
\end{aligned}
$$

Ainsi dans tous les cas, $f^{\prime}\left(L_{0}, \ldots, L_{u}\right)$ s'obtient en spécialisant chaque variable de $f$ en un élément de la forme $a X_{0}+b X_{i}$ avec $a, b \in \mathbb{Z},|a|,|b| \leq \frac{1}{2} \max (D, 2)$ et $1 \leq i \leq u+1$. Par conséquent, nous pouvons écrire

$$
P=X_{0}^{-D u} \sum_{\mathfrak{m}} \lambda_{\mathfrak{m}} \prod_{i=1}^{u+1} \prod_{k=1}^{D}\left(a_{\mathfrak{m}, i, k} X_{0}+b_{\mathfrak{m}, i, k} X_{i}\right)
$$

si les $\lambda_{\mathfrak{m}}$ sont les coefficients de $f, \mathfrak{m}$ parcourant les monômes unitaires de multidegré $d=(D, \ldots, D)$. Si $v$ est une place finie de $\mathbb{Q}\left(\left(\lambda_{\mathfrak{m}}\right)_{\mathfrak{m}}\right)$, la valeur absolue d'un coefficient de $P$ est au plus $\max _{\mathfrak{m}}\left|\lambda_{\mathfrak{m}}\right|_{v}$ (car $a_{\mathfrak{m}, i, k}$ et $b_{\mathfrak{m}, i, k}$ sont entiers). Pour une place infinie, on remarque que le terme en facteur de $\lambda_{\mathfrak{m}}$ est un polynôme dont la somme des valeurs absolues des coefficients est au plus $D^{D(u+1)}$ (y compris si $D=1$ ). Par ailleurs,

$$
\left|\lambda_{\mathfrak{m}}\right|_{v} \leq\left(\begin{array}{c}
d \\
\mathfrak{m}
\end{array}\right) M_{v}(f)
$$

si $M_{v}(f)$ est le terme local définissant $h_{\varphi}(Z)$ (voir la démonstration du lemme 3.3 dans [10]). Ainsi

$$
|P|_{v} \leq D^{D(u+1)} \sum_{\mathfrak{m}}\left(\begin{array}{l}
d \\
\mathfrak{m}
\end{array}\right) M_{v}(f)=[D(n+1)]^{D(u+1)} M_{v}(f)
$$

et la majoration de $h(B)$ en découle bien.

TOME $133-2005-\mathrm{N}^{\mathrm{O}} 4$ 
2.2. Réduction de polynômes. - Nous utiliserons plusieurs fois un procédé de réduction de polynômes modulo certaines relations de la forme de celles obtenues au lemme 2.3. Nous donnons ici le résultat technique (mais élémentaire) après un lemme préparatoire.

LEMME 2.4. - On peut associer à chaque triplet $(x, y, z) \in \mathbb{N}^{3}$ avec $1 \leq y \leq z$ un polynôme $U_{x, y, z} \in \mathbb{N}\left[X_{1}, \ldots, X_{z}\right]$, homogène de degré $x+y-z$ si $\operatorname{deg} X_{i}=i$ et dont la somme des coefficients est au plus $2^{x}$, de telle sorte que, si, dans un anneau $A$, des éléments $t, a_{1}, \ldots, a_{z}$ vérifient

$$
t^{z}=\sum_{y=1}^{z} a_{y} t^{z-y}
$$

alors, pour tout $x \in \mathbb{N}$,

$$
t^{x}=\sum_{y=1}^{z} U_{x, y, z}\left(a_{1}, \ldots, a_{z}\right) t^{z-y}
$$

Démonstration. - On travaille d'abord dans l'anneau $A=\mathbb{Z}\left[X_{1}, \ldots, X_{z}, T\right]$ gradué par $\operatorname{deg} X_{i}=i$ et $\operatorname{deg} T=1$. Le polynôme $P=T^{z}-\sum_{y=1}^{z} X_{y} T^{z-y}$ est homogène; on note $t$ l'image de $T$ dans $A / P$ qui est gradué. En écrivant $t^{x}$ dans la $\mathbb{Z}$-base évidente de $A / P$ on obtient immédiatement des $U_{x, y, z}$ à coefficients dans $\mathbb{Z}$ ayant la bonne homogénéité. En substituant directement il est clair que les coefficients sont positifs. Enfin on considère $A=\mathbb{R}$. Il est immédiat qu'il existe $t$ dans l'intervalle $\left[1,2\left[\right.\right.$ tel que $t^{z}=\sum_{y=1}^{z} t^{z-y}$. Par suite, pour tout $x$,

$$
2^{x} \geq t^{x}=\sum_{y=1}^{z} U_{x, y, z}(1, \ldots, 1) t^{z-y} \geq \sum_{y=1}^{z} U_{x, y, z}(1, \ldots, 1) .
$$

Par positivité, la somme des coefficients de $U_{x, y, z}$ est bien $U_{x, y, z}(1, \ldots, 1)$.

Voici le résultat qui permet le contrôle de la hauteur des réductions.

Lemme 2.5. - Soit $m$ un entier naturel. Pour chaque entier $i$ entre 1 et $m$, considérons des variables $V_{0}^{(i)}, \ldots, V_{u_{i}}^{(i)}$ et $W_{0}^{(i)}, \ldots, W_{n_{i}}^{(i)}$ (pour certains $\left.u_{i}, n_{i} \geq 0\right)$ ainsi que pour chaque entier $j$ entre 0 et $n_{i}$, un polynôme $P_{j}^{(i)} \in \overline{\mathbb{Q}}\left[V_{0}^{(i)}, \ldots, V_{u_{i}}^{(i)}, W_{j}^{(i)}\right]$ homogène de degré $d_{i, j} \geq 1$ de sorte que le coefficient de $W_{j}^{(i)^{d_{i, j}}}$ soit égal à 1 . Soit $B_{i}$ la famille des coefficients des $P_{0}^{(i)}, \ldots, P_{n_{i}}^{(i)}$ et notons $\mathcal{K}=\left\{\left(k_{i, j}\right)_{1 \leq i \leq m, 0 \leq j \leq n_{i}} ; 0 \leq k_{i, j}<d_{i, j}\right\}$.

Si $\mathcal{S}$ est un ensemble fini, $b$ un élément de $\mathbb{N}^{m}$ et $\left(\Psi_{s}\right)_{s \in \mathcal{S}}$ une famille de polynômes multihomogènes en $W$ de multidegré $b$, alors il existe une unique famille $\left(Q_{k, s}\right)_{(k, s) \in \mathcal{K} \times \mathcal{S}}$ de polynômes multihomogènes en $V$ telle que, pour tout $s \in \mathcal{S}$,

$$
\Psi_{s}-\sum_{k \in \mathcal{K}} Q_{k, s}(V) W^{k}
$$

BULlETiN DE LA SOCIÉtÉ MATHÉMATIQUE DE FRANCE 
est élément de l'idéal $J$ engendré par tous les $P_{j}^{(i)}$. De plus, les coefficients de $Q_{k, s}$ appartiennent au corps engendré par les coefficients de $\Psi_{s}$ et les $B_{i}$ et

$$
h\left(\left(Q_{k, s}\right)_{k, s}\right) \leq h\left(\left(\Psi_{s}\right)_{s}\right)+\sum_{i=1}^{m} b_{i}\left(h\left(B_{i}\right)+\log \left(2 u_{i}+2\right)\right)+\log \left(\begin{array}{c}
b_{i}+n_{i} \\
n_{i}
\end{array}\right) .
$$

Démonstration. — Écrivons

$$
P_{j}^{(i)}=W_{j}^{(i)^{d_{i, j}}}-\sum_{y=1}^{d_{i, j}} P_{j, y}^{(i)}\left(V^{(i)}\right) W_{j}^{(i)^{d_{i, j}-y}}
$$

de sorte que par le lemme précédent (appliqué à $\overline{\mathbb{Q}}\left[V^{(i)}, W_{j}^{(i)}\right] / P_{j}^{(i)}$ ) l'élément (homogène de degré $\ell$ )

$$
W_{j}^{(i)}{ }^{\ell}-\sum_{y=1}^{d_{i, j}} U_{\ell, y, d_{i, j}}\left(P_{j, 1}^{(i)}, \ldots, P_{j, d_{i, j}}^{(i)}\right) W_{j}^{(i)^{d_{i, j}-y}}
$$

appartient à $J$. Par ailleurs notons

$$
\Psi_{s}=\sum_{k^{\prime} \in \mathcal{K}^{\prime}} \lambda_{k^{\prime}, s} W^{k^{\prime}}
$$

où $\mathcal{K}^{\prime}=\left\{\left(k_{i, j}^{\prime}\right)_{1 \leq i \leq m, 0 \leq j \leq n_{i}} ; \sum_{j=0}^{n_{i}} k_{i, j}^{\prime}=b_{i}\right.$ pour tout $\left.i\right\}$. Nous pouvons alors choisir

$$
Q_{k, s}(V)=\sum_{k^{\prime} \in \mathcal{K}^{\prime}} \lambda_{k^{\prime}, s} \prod_{i=1}^{m} \prod_{j=1}^{n_{i}} U_{k_{i, j}^{\prime}, d_{i, j}-k_{i, j}, d_{i, j}}\left(P_{j, 1}^{(i)}, \ldots, P_{j, d_{i, j}}^{(i)}\right) .
$$

L'unicité est claire pour raison de degré. Il reste à estimer la hauteur. Le polynôme $U_{k_{i, j}^{\prime}, d_{i, j}-k_{i, j}, d_{i, j}}\left(P_{j, 1}^{(i)}, \ldots, P_{j, d_{i, j}}^{(i)}\right)$ est une somme d'au plus $2^{k_{i, j}^{\prime}}$ termes de la forme

$$
\prod_{y=1}^{d_{i, j}} P_{j, y}^{(i)^{\ell_{y}}} \quad \text { où } \quad \sum_{y=1}^{d_{i, j}} y \ell_{y}=k_{i, j}^{\prime}-k_{i, j}
$$

Un tel terme est à son tour somme d'au plus $\prod_{y=1}^{d_{i, j}}\left(\begin{array}{c}u_{i}+y \\ y\end{array}\right)^{\ell_{y}}$ termes (puisque $\left.\operatorname{deg} P_{j, y}^{(i)}=y\right)$, de la forme

$$
\prod_{\alpha=1}^{k_{i, j}^{\prime}-k_{i, j}} \beta_{\alpha} V_{\nu_{\alpha}}^{(i)}
$$

où $\beta_{\alpha} \in B_{i}$ (on rappelle $1 \in B_{i}$ ) et $0 \leq \nu_{\alpha} \leq u_{i}$. Nous majorons

$$
2^{k_{i, j}^{\prime}} \prod_{y=1}^{d_{i, j}}\left(\begin{array}{c}
u_{i}+y \\
y
\end{array}\right)^{\ell_{y}} \leq 2^{k_{i, j}^{\prime}} \prod_{y=1}^{d_{i, j}}\left(u_{i}+1\right)^{y \ell_{y}} \leq\left(2 u_{i}+2\right)^{k_{i, j}^{\prime}} .
$$

TOME $133-2005-\mathrm{N}^{\mathrm{O}} 4$ 
Sachant que $\sum_{j} k_{i, j}^{\prime}=b_{i}$, nous trouvons que le polynôme $Q_{k, s}$ s'exprime comme une somme d'au plus

$$
\operatorname{Card}\left(\mathcal{K}^{\prime}\right) \prod_{i=1}^{m}\left(2 u_{i}+2\right)^{b_{i}}=\prod_{i=1}^{m}\left(\begin{array}{c}
b_{i}+n_{i} \\
n_{i}
\end{array}\right)\left(2 u_{i}+2\right)^{b_{i}}
$$

termes de la forme

$$
\lambda_{k^{\prime}, s} \prod_{i=1}^{m} \prod_{\alpha=1}^{b_{i}-\Sigma_{j} k_{i, j}} \beta_{\alpha, i} V_{\nu_{\alpha, i}}^{(i)}
$$

où $\beta_{\alpha, i} \in B_{i}$ et $0 \leq \nu_{\alpha, i} \leq u_{i}$. La majoration de la hauteur s'en déduit immédiatement.

Citons également ici la version que nous emploierons du très classique lemme de Siegel.

Lemme 2.6 (lemme de Siegel). - Pour tout corps de nombres $K$, il existe une constante $c_{S}(K)$ telle que pour toute matrice $A \in \mathcal{M}_{m \times n}(K)$ avec $m<n$, il existe $X \in K^{n} \backslash\{0\}$ vérifiant $A X=0$ et

$$
h(X) \leq \frac{m}{n-m}\left(\max _{1 \leq i \leq m} h\left(A_{i}\right)+\log n\right)+\frac{n}{n-m} c_{S}(K) .
$$

Démonstration. — C'est le lemme de Siegel pour un corps de nombres tel qu'il est formulé dans [1] (il est en fait possible, avec le lemme de Bombieri-Vaaler, de supprimer le coefficient $n /(n-m)$ devant la constante, mais ce raffinement n'a pas d'intérêt ici).

2.3. Lemme local. — Nous avons besoin de généraliser le lemme 5 de [2] pour estimer localement les dérivées successives d'un élément algébrique sur un anneau de polynômes.

Soient $K$ un corps de nombres, $u$ un entier $\geq 1$ et $X_{1}, \ldots, X_{u}, Y$ des indéterminées. Nous considérons un polynôme $P \in \bar{K}[X, Y]=K\left[X_{1}, \ldots, X_{u}, Y\right]$ qui s'écrive $P=P_{1}^{b}$ avec $b \geq 1$ et $P_{1} \in K[X, Y]$ irréductible. Soient encore $L$ une extension algébrique de $K(X)$ et $y \in L$ un élément tel que $P(X, y)=0$. Notons $\left(\mathrm{d}_{i}: L \rightarrow L\right)_{1 \leq i \leq u}$ les $K$-dérivations caractérisées par $\mathrm{d}_{i}\left(X_{j}\right)=\delta_{i, j}$ puis, lorsque $\kappa \in \mathbb{N}^{u}$,

$$
\mathrm{d}^{\kappa}=\mathrm{d}_{1}^{\kappa_{1}} \circ \ldots \circ \mathrm{d}_{u}^{\kappa_{u}}, \quad \kappa !=\kappa_{1} ! \ldots \kappa_{u} ! \quad \text { et } \quad \partial^{\kappa}=\frac{1}{\kappa !} \mathrm{d}^{\kappa} .
$$

Finalement, pour une place $v$ de $K$, nous écrivons $\varepsilon_{v}=1$ si $v$ est infinie et $\varepsilon_{v}=0$ sinon.

Nous pouvons alors donner l'estimation suivante.

LEMme 2.7. - Soit $\varphi: K[X, y] \rightarrow K$ un morphisme de K-algèbres tel que $\varphi\left(X_{i}\right)=0$ pour $1 \leq i \leq u$ et notons encore $\varphi$ son extension lorsqu'on localise en $\operatorname{Ker}(\varphi)$. Soit $R=b !^{-1}\left(\partial^{b} P / \partial Y^{b}\right)$. Nous supposons $\varphi(R(X, y)) \neq 0$. Soient 
ensuite $v$ une place de $K, a \in K^{\times}$tel que $|\varphi(y)|_{v} \leq|a|_{v}$ et $\kappa \in \mathbb{N}^{u} \backslash\{0\}$. Alors nous avons

$$
\left|\varphi\left(\partial^{\kappa} y\right)\right|_{v} \leq|a|_{v} f_{1}(u, D)^{\varepsilon_{v}|\kappa|}\left(\frac{|P(X, a Y)|_{v}}{\left|a^{b} \varphi(R(X, y))\right|_{v}}\right)^{(2|\kappa|-1) / b}
$$

où $D$ majore à la fois $\operatorname{deg}_{X} P, \operatorname{deg}_{Y} P$ et

$$
f_{1}(u, D)=(2 u+4)^{1+\frac{3}{2} D} D\left(\begin{array}{c}
D+u \\
u
\end{array}\right)^{2}\left(\begin{array}{c}
D+1 \\
2
\end{array}\right)^{2} \text {. }
$$

Démonstration. - Commençons par remarquer qu'en remplaçant $y$ par $y / a$ et $P$ par le polynôme $P(X, a Y)$, il suffit de démontrer l'énoncé pour $a=1$. Nous allons également supposer $b=1$ dans un premier temps. Nous adaptons dans ce cas la preuve de [2] (qui correspond au cas $u=1$ ). Notons

$$
\chi: K\left[X_{1}, \ldots, X_{u}, Y\right] \longrightarrow K\left[X_{1}, \ldots, X_{u}, y\right] \text { et } \psi=\varphi \circ \chi
$$

L'écriture $\varphi\left(\partial^{\kappa} y\right)$ a bien un sens car le localisé $K\left[X_{1}, \ldots, X_{u}, y\right]_{\operatorname{Ker}(\varphi)}$ est stable par chaque $\mathrm{d}_{i}$ : en effet $\mathrm{d}_{i} y=-\chi(R)^{-1} \chi\left(\partial P / \partial X_{i}\right)$. Écrivons maintenant

$$
P=\sum_{i, j} p_{i, j} X_{1}^{j_{1}} \cdots X_{u}^{j_{u}} Y^{i}
$$

et distinguons selon que $v$ est une place finie ou infinie.

Dans le premier cas, nous tirons parti de la formule de Leibniz pour avoir

$$
0=\partial^{\kappa}(\chi(P))=\sum_{i, j} p_{i, j} \sum_{\ell^{(0)}+\cdots+\ell^{(i)}=\kappa} \partial^{\ell^{(0)}}\left(X_{1}^{j_{1}} \cdots X_{u}^{j_{u}}\right) \partial^{\ell^{(1)}} y \cdots \partial^{\ell^{(i)}} y
$$

où la seconde somme est sur tous les choix de $\ell^{(0)}, \ldots, \ell^{(i)} \in \mathbb{N}^{u}$ qui vérifient $\ell^{(0)}+\cdots+\ell^{(i)}=\kappa$. Par ailleurs $\chi(R)=\sum_{i, j} p_{i, j} X_{1}^{j_{1}} \cdots X_{u}^{j_{u}} i y^{i-1}$; donc, en extrayant de la somme précédente les termes pour lesquels $\ell^{(k)}=\kappa$ pour un indice $k$ vérifiant $0<k \leq i$, nous obtenons

$$
\chi(R) \partial^{\kappa} y=-\sum_{i, j} p_{i, j} \sum_{\substack{\ell^{(0)}+\cdots+\ell^{(i)}=\kappa \\ \ell^{(k)} \neq \kappa \text { si } k>0}} \partial^{\ell^{(0)}}\left(X_{1}^{j_{1}} \cdots X_{u}^{j_{u}}\right) \partial^{\ell^{(1)}} y \cdots \partial^{\ell^{(i)}} y .
$$

La quantité $\varphi\left(\partial^{\ell^{(0)}}\left(X_{1}^{j_{1}} \cdots X_{u}^{j_{u}}\right)\right)$ vaut 1 si $\ell^{(0)}=j$ et 0 sinon. Nous appliquons alors $\varphi$ à l'égalité précédente et, $v$ étant ultramétrique, il vient

$$
|\psi(R)|_{v} \cdot\left|\varphi\left(\partial^{\kappa} y\right)\right|_{v} \leq|P|_{v} \max _{\substack{\ell^{(1)}+\cdots+\ell^{(i)} \leq \kappa \\ i \geq 0, \ell^{(k)} \neq \kappa}}\left|\varphi\left(\partial^{\ell^{(1)}} y\right)\right|_{v} \cdots\left|\varphi\left(\partial^{\ell^{(i)}} y\right)\right|_{v} .
$$

Ceci permet de montrer par récurrence sur $|\kappa|$ que

$$
\left|\varphi\left(\partial^{\kappa} y\right)\right|_{v} \leq\left(\frac{|P|_{v}}{|\psi(R)|_{v}}\right)^{\max (0,2|\kappa|-1)} .
$$

TOME $133-2005-\mathrm{N}^{\mathrm{O}} 4$ 
En effet, le cas $|\kappa|=0$ est l'hypothèse $|\varphi(y)|_{v} \leq 1$ et, pour $|\kappa|>0$, la formule obtenue montre que, si l'hypothèse de récurrence est vérifiée pour les $\kappa^{\prime}$ avec $\left|\kappa^{\prime}\right|<|\kappa|$, nous avons

$$
\left|\varphi\left(\partial^{\kappa} y\right)\right|_{v} \leq \max _{\substack{\ell^{(1)}+\cdots+\ell^{(i)} \leq \kappa \\ i \geq 0, \ell^{(k)} \neq \kappa}}\left(\frac{|P|_{v}}{|\psi(R)|_{v}}\right)^{1+\sum_{k=1}^{i} \max \left(0,2\left|\ell^{(k)}\right|-1\right)} .
$$

Or $|\psi(R)|_{v} \leq|P|_{v}$ et par ailleurs

$$
\sum_{k=1}^{i} \max \left(0,2\left|\ell^{(k)}\right|-1\right) \leq 2|\kappa|-2
$$

pour tous les choix de $i \geq 0, \ell^{(1)}, \ldots, \ell^{(i)} \in \mathbb{N}^{u}$ avec $\ell^{(1)}+\cdots+\ell^{(i)} \leq \kappa$ et $\ell^{(k)} \neq \kappa$. Ceci termine la démonstration quand $v$ est une place finie (et $\bar{b}=1$ ).

Nous procédons différemment lorsque $v$ est infinie. Montrons d'abord par récurrence sur $|\kappa| \geq 1$ qu'il existe un polynôme $Q_{\kappa} \in K\left[X_{1}, \ldots, X_{u}, Y\right]$ tel que

$$
\chi\left(Q_{\kappa}\right)=\chi(R)^{2|\kappa|-1} \mathrm{~d}^{\kappa}(y) .
$$

En effet si $|\kappa|=1$, c'est-à-dire $\kappa_{k}=\delta_{k, k_{0}}$ pour un indice $k_{0}$, il est loisible de poser $Q_{\kappa}=-\partial P / \partial X_{k_{0}}$ car

$$
0=\mathrm{d}^{\kappa}(\chi(P))=\chi\left(\frac{\partial P}{\partial X_{k_{0}}}\right) \mathrm{d}^{\kappa}\left(X_{k_{0}}\right)+\chi\left(\frac{\partial P}{\partial Y}\right) \mathrm{d}^{\kappa}(y)=\chi\left(-Q_{\kappa}\right)+\chi(R) \mathrm{d}^{\kappa}(y) .
$$

Si $|\kappa|>1$, choisissons $k_{0}$ tel que $\kappa_{k_{0}} \neq 0$ et définissons $\kappa^{\prime}$ par $\kappa_{k}^{\prime}=\kappa_{k}-\delta_{k, k_{0}}$ puis

$$
Q_{\kappa}=R^{2} \frac{\partial Q_{\kappa^{\prime}}}{\partial X_{k_{0}}}-R \frac{\partial P}{\partial X_{k_{0}}} \frac{\partial Q_{\kappa^{\prime}}}{\partial Y}+\left(2\left|\kappa^{\prime}\right|-1\right) Q_{\kappa^{\prime}}\left(\frac{\partial P}{\partial X_{k_{0}}} \frac{\partial R}{\partial Y}-R \frac{\partial R}{\partial X_{k_{0}}}\right) .
$$

Ceci convient car en appliquant $\mathrm{d}_{k_{0}}$ à $\chi(R)^{2\left|\kappa^{\prime}\right|-1} \mathrm{~d}^{\kappa^{\prime}} y=\chi\left(Q_{\kappa^{\prime}}\right)$ puis en multipliant par $\chi(R)^{2}$, il vient

$$
\begin{aligned}
\chi(R)^{2|\kappa|-1} & \mathrm{~d}^{\kappa} y+\left(2\left|\kappa^{\prime}\right|-1\right) \chi(R)^{2\left|\kappa^{\prime}\right|} \mathrm{d}_{k_{0}}(\chi(R)) \mathrm{d}^{\kappa^{\prime}} y \\
= & \chi\left(\frac{\partial Q_{\kappa^{\prime}}}{\partial X_{k_{0}}}\right) \chi(R)^{2}+\chi\left(\frac{\partial Q_{\kappa^{\prime}}}{\partial Y}\right) \chi(R)^{2} \mathrm{~d}_{k_{0}} y
\end{aligned}
$$

soit

$$
\begin{gathered}
\chi(R)^{2|\kappa|-1} \mathrm{~d}^{\kappa} y+\left(2\left|\kappa^{\prime}\right|-1\right) \chi\left(Q_{\kappa^{\prime}} R\right)\left(\chi\left(\frac{\partial R}{\partial Y}\right) \mathrm{d}_{k_{0}} y+\chi\left(\frac{\partial R}{\partial X_{k_{0}}}\right)\right) \\
=\chi\left(\frac{\partial Q_{\kappa^{\prime}}}{\partial X_{k_{0}}} R^{2}\right)+\chi\left(\frac{\partial Q_{\kappa^{\prime}}}{\partial Y}\right) \chi(R)^{2} \mathrm{~d}_{k_{0}} y
\end{gathered}
$$

formule dans laquelle il suffit de remplacer $\chi(R) \mathrm{d}_{k_{0}} y$ par $-\chi\left(\partial P / \partial X_{k_{0}}\right)$ pour reconnaître

$$
\chi(R)^{2|\kappa|-1} \mathrm{~d}^{\kappa} y=\chi\left(Q_{\kappa}\right) .
$$

BULLETIN DE LA SOCIÉTÉ MATHÉMATIQUE DE FRANCE 
Sur la définition de $Q_{\kappa}$ par récurrence, il est clair que $\operatorname{deg}_{X} Q_{\kappa}$ et $\operatorname{deg}_{Y} Q_{\kappa}$ sont tous deux majorés par $2|\kappa| D$. D'autre part, si $Q_{\kappa^{\prime}}=\sum_{\ell, m} q_{\ell, m} X_{1}^{m_{1}} \cdots X_{u}^{m_{u}} Y^{\ell}$, cette définition donne

$$
\begin{aligned}
Q_{\kappa}= & \sum_{i, j, i^{\prime}, j^{\prime}, \ell, m} p_{i, j} p_{i^{\prime}, j^{\prime}} q_{\ell, m} X^{j+j^{\prime}+m} X_{k_{0}}^{-1} Y^{i+i^{\prime}+\ell-2} \\
& \times\left(i i^{\prime} m_{k_{0}}-i j_{k_{0}}^{\prime} \ell+\left(2\left|\kappa^{\prime}\right|-1\right)\left(j_{k_{0}}^{\prime} i(i-1)-i i^{\prime} j_{k_{0}}^{\prime}\right)\right) .
\end{aligned}
$$

Dans cette expression, nous avons

$$
\left|i i^{\prime}\left(m_{k_{0}}-\left(2\left|\kappa^{\prime}\right|-1\right) j_{k_{0}}^{\prime}\right)\right| \leq i i^{\prime} \max \left(m_{k_{0}},\left(2\left|\kappa^{\prime}\right|-1\right) j_{k_{0}}^{\prime}\right) \leq i i^{\prime} \cdot 2\left|\kappa^{\prime}\right| D
$$

et de même $\left|i j_{k_{0}}^{\prime}\left(-\ell+\left(2\left|\kappa^{\prime}\right|-1\right)(i-1)\right)\right| \leq i j_{k_{0}}^{\prime} \cdot 2\left|\kappa^{\prime}\right| D$. Par suite

$$
\left|Q_{\kappa}\right|_{v} \leq|P|_{v}^{2} \cdot\left|Q_{\kappa^{\prime}}\right|_{v} \cdot 2\left|\kappa^{\prime}\right| D \sum_{0 \leq i, i^{\prime},|j|,\left|j^{\prime}\right| \leq D} i\left(i^{\prime}+j_{k_{0}}^{\prime}\right) .
$$

Cette dernière somme vaut

$$
\left(\begin{array}{c}
D+u \\
u
\end{array}\right)^{2}\left(\begin{array}{c}
D+1 \\
2
\end{array}\right)^{2}\left[1+\frac{2}{u+1}\right] \leq \frac{f_{1}^{\prime}(u, D)}{2 u D}
$$

si nous notons $f_{1}^{\prime}(u, D)=f_{1}(u, D)(2 u+4)^{-\frac{3}{2} D}$. Ainsi

$$
\left|Q_{\kappa}\right|_{v} \leq f_{1}^{\prime}(u, D) \frac{\left|\kappa^{\prime}\right|}{u}|P|_{v}^{2} \cdot\left|Q_{\kappa^{\prime}}\right|_{v} .
$$

Si $|\kappa|=1$, on a $\left|Q_{\kappa}\right|_{v} \leq D|P|_{v}$ donc

$$
\left|Q_{\kappa}\right|_{v} \leq D\left(\frac{f_{1}^{\prime}(u, D)}{u}\right)^{|\kappa|-1}(|\kappa|-1) !|P|_{v}^{2|\kappa|-1} .
$$

S'en déduit la majoration

$$
\left|\psi\left(Q_{\kappa}\right)\right|_{v} \leq 3 D^{2}|\kappa| !\left(\frac{f_{1}^{\prime}(u, D)}{u}\right)^{|\kappa|-1}|P|_{v}^{2|\kappa|-1}
$$

$\operatorname{car}|\psi(Y)|_{v} \leq 1, \psi\left(X_{i}\right)=0$ et $1+\operatorname{deg}_{Y} Q_{\kappa} \leq 3 D|\kappa|$. Enfin le choix de $Q_{\kappa}$ montre que

$$
\psi\left(Q_{\kappa}\right)=\psi(R)^{2|\kappa|-1} \varphi\left(\mathrm{d}^{\kappa} y\right)=\kappa_{1} ! \ldots \kappa_{u} ! \psi(R)^{2|\kappa|-1} \varphi\left(\partial^{\kappa} y\right)
$$

donc

$$
\begin{aligned}
\left|\varphi\left(\partial^{\kappa} y\right)\right|_{v} & \leq \frac{|\kappa| !}{\kappa !} 3 D^{2}\left(\frac{f_{1}^{\prime}(u, D)}{u}\right)^{|\kappa|-1}\left(\frac{|P|_{v}}{|\psi(R)|_{v}}\right)^{2|\kappa|-1} \\
& \leq f_{1}^{\prime}(u, d)^{|\kappa|}\left(\frac{|P|_{v}}{|\psi(R)|_{v}}\right)^{2|\kappa|-1}
\end{aligned}
$$

$\operatorname{car}|\kappa| ! / \kappa ! \leq u^{|\kappa|}$ et $3 u D^{2} \leq f_{1}^{\prime}(u, D)$.

Le cas $b=1$ est donc entièrement établi avec le gain d'un facteur égal à $(2 u+4)^{\frac{3}{2} \varepsilon_{v} D|\kappa|}$. Dans le cas général, nous pouvons appliquer ce qui précède au polynôme $P_{1}$ parce que, si $R_{1}=\partial P_{1} / \partial Y$, nous avons $R=R_{1}^{b}+P_{1} Q$ avec un

TOME $133-2005-\mathrm{N}^{\mathrm{O}} 4$ 
certain polynôme $Q$ et donc $R(X, y)=R_{1}(X, y)^{b}$. Il reste seulement à voir que l'on a

Comme $b \geq 2$, ceci découle de

$$
\left|P_{1}\right|_{v}^{2|\kappa|-1} \leq(2 u+4)^{\frac{3}{2} \varepsilon_{v} D|\kappa|}|P|_{v}^{(2|\kappa|-1) / b} .
$$

$$
\left|P_{1}\right|_{v} \leq(2 u+2)^{\frac{3}{2} \varepsilon_{v} D / b}|P|_{v}^{1 / b},
$$

cette dernière inégalité s'obtenant en notant que $P_{1}$ est de degré au plus $D / b$ en chacun des deux groupes de variables (pour une place infinie, on procède comme dans [7, chap. 3, prop. 2.12] en transitant par la mesure de Mahler).

\section{Réduction du problème}

Nous nous plaçons désormais sous les hypothèses du théorème 1.2.

Considérons l'ensemble $\mathcal{E}$ des $m$-uplets $\left(Y_{1}, \ldots, Y_{m}\right)$ formés de sous-schémas fermés intègres de $X$ tels que si nous notons $u_{i}=\operatorname{dim} Y_{i}, u=u_{1}+\cdots+u_{m}$ et $D_{i}=\operatorname{deg} Y_{i}$ pour $1 \leq i \leq m$, alors

$$
\begin{aligned}
& x_{i} \in Y_{i}(\overline{\mathbb{Q}}) \quad \text { pour } 1 \leq i \leq m, \\
& D_{i} \leq(\operatorname{deg} X) \Lambda^{\psi(u)-1} \quad \text { pour } 1 \leq i \leq m, \\
& \prod_{i=1}^{m} D_{i} \leq(\operatorname{deg} X)^{m} \Lambda^{\psi(u)-1} \quad \text { et } \\
& \sum_{i=1}^{m} a_{i} h\left(Y_{i}\right) \leq \frac{1}{2} \Lambda^{2 \psi(u)}\left(M t_{2}\right)^{m \operatorname{dim} X-u}|a| \max (h(X), \delta) .
\end{aligned}
$$

En premier lieu, il est clair que $(X, \ldots, X) \in \mathcal{E}$ car $\psi(m \operatorname{dim} X)=1$ et $\Lambda^{2} \geq 2$. Par conséquent, il existe un élément $\left(Y_{1}, \ldots, Y_{m}\right)$ de $\mathcal{E}$ pour lequel la dimension $u$ est minimale. Nous allons travailler avec un tel élément que nous fixons dorénavant. Il faut remarquer que $u$ n'est pas nul : en effet, si c'était le cas, nous aurions nécessairement $Y_{i}=\left\{x_{i}\right\}$ pour $1 \leq i \leq m$ et nous pourrions donc déduire de la dernière inégalité

$$
\begin{aligned}
|a| c_{3} & \leq \sum_{i=1}^{m} a_{i} h\left(x_{i}\right) \leq \sum_{i=1}^{m} a_{i} h\left(\left\{x_{i}\right\}\right) \\
& \leq \frac{1}{2} \Lambda^{2 \psi(0)}\left(M t_{2}\right)^{m \operatorname{dim} X}|a| \max (h(X), \delta)=\frac{1}{2}|a| c_{3},
\end{aligned}
$$

ce qui est faux.

La démonstration consistera à montrer que si la conclusion du théorème est en défaut alors il est possible de construire un élément de $\mathcal{E}$ de dimension $u-1$. Pour faire cette construction générale, il est commode d'une part de modifier le plongement $\iota$ sur chaque facteur pour que $Y_{i}$ soit décrit de manière simple et d'autre part d'exclure de la construction un certain nombre de cas dégénérés (en construisant directement un élément de $\mathcal{E}$ dans ces cas-là).

BULletin DE LA SOCiÉtÉ MATHÉmATiQUe DE FRANCE 
Nous appliquons donc les résultats du premier paragraphe de la partie précédente aux immersions fermées $\varphi_{i}: Y_{i} \hookrightarrow \mathbb{P}_{\overline{\mathbb{Q}}}^{N}$ déduites de $\iota$ (pour $1 \leq i \leq m$ ).

Ainsi, en vertu de la proposition 2.2, il existe pour chaque $i$ une matrice $M_{i} \in$ $\mathrm{GL}_{N+1}(\overline{\mathbb{Q}})$ à coefficients entiers compris entre $-\max \left(1, \frac{1}{2} D_{i}\right)$ et $\max \left(1, \frac{1}{2} D_{i}\right)$ de sorte que le morphisme associé $\chi_{i}: \mathbb{P}_{\overline{\mathbb{Q}}}^{N} \rightarrow \mathbb{P}_{\overline{\mathbb{Q}}}^{N}$ fait de $\chi_{i} \circ \varphi_{i}$ un plongement adapté. Nous fixons une fois pour toutes de telles matrices $M_{1}, \ldots, M_{m}$ ainsi que des polynômes $P_{j}^{(i)}$ et $Q_{j}^{(i)}(1 \leq i \leq m$ et $0 \leq j \leq N)$ données par le lemme 2.3. Nous notons $B_{i}$ la famille de leurs coefficients pour $i$ fixé.

Pour distinguer les différentes coordonnées de $\left(\mathbb{P}_{\overline{\mathbb{Q}}}^{N}\right)^{m}$, nous désignons celles du $i$-ième facteur par $W_{j}^{(i)}(0 \leq j \leq N)$ à la source de $\chi_{i}$ et $V_{j}^{(i)}(0 \leq j \leq N)$ à son but. Par exemple, le polynôme de $Q_{j}^{(i)}\left(V_{0}^{(i)}, \ldots, V_{u_{i}}^{(i)}, W_{j}^{(i)}\right)$ est élément de l'idéal de $\chi_{i} \circ \varphi_{i}\left(Y_{i}\right)$.

Nous notons également $w_{j}^{(i)}$ et $v_{j}^{(i)}$ les images de $W_{j}^{(i)} / V_{0}^{(i)}$ et $V_{j}^{(i)} / V_{0}^{(i)}$ dans le corps des fonctions $K\left(Y_{i}\right)$ (de sorte qu'en particulier ces éléments sont liés par la relation matricielle $\left.\left(v^{(i)}\right)=M_{i}\left(w^{(i)}\right)\right)$. Finalement, le morphisme fini $Y_{i} \rightarrow \mathbb{P}_{K}^{u_{i}}$ défini par $V_{0}^{(i)}, \ldots, V_{u_{i}}^{(i)}$ s'appellera $\rho_{i}$ et $b_{i, j}$ sera l'entier tel que $Q_{j}^{(i)}$ est égal à un polynôme irréductible élevé à la puissance $b_{i, j}$.

Dans le cadre ainsi fixé, nous pouvons donner l'énoncé par lequel passe la preuve du théorème 1.2 par l'absurde.

Proposition 3.1. - Il n'existe pas de couple $(\ell, U)$ avec $1 \leq \ell \leq m$ et $U$ un polynôme homogène de $\overline{\mathbb{Q}}\left[V_{0}^{(\ell)}, \ldots, V_{u_{\ell}}^{(\ell)}\right]$ tel que

1) $\chi_{\ell}\left(x_{\ell}\right) \in V(U)$,

2) $\chi_{\ell}\left(Y_{\ell}\right) \not \subset V(U)$

3) $\operatorname{deg} U \leq \Lambda^{\omega^{\prime} u \psi(u)}$ et

4) $a_{\ell} h(U) \leq \frac{|a|}{8 \operatorname{deg} X} \Lambda^{\omega^{\prime}(u+1) \psi(u)+m-1-\omega}\left(M t_{2}\right)^{m \operatorname{dim} X-u+1} \max (h(X), \delta)$.

Démonstration. - Si un tel couple existe, nous définissons $Y_{i}^{\prime}=Y_{i}$ si $i \neq \ell$ et $Y_{\ell}^{\prime}$ comme une composante irréductible de $Y_{\ell} \cap \chi_{\ell}^{-1}(V(U))$ contenant $x_{\ell}$. Il nous suffit alors de montrer que $\left(Y_{1}^{\prime}, \ldots, Y_{m}^{\prime}\right)$ appartient à $\mathcal{E}$.

Dans un premier temps, écrivons $\chi_{\ell}^{-1}(V(U))=V\left(U^{\prime}\right)$ où $U^{\prime}$ est l'élément de $\overline{\mathbb{Q}}\left[W_{0}^{(\ell)}, \ldots, W_{N}^{(\ell)}\right]$ défini par

$$
U^{\prime}\left(W^{(\ell)}\right)=U\left(M_{\ell} \cdot W^{(\ell)}\right) .
$$

Il vient $\operatorname{deg} U^{\prime}=\operatorname{deg} U$ et, vu la majoration des coefficients de $M_{\ell}$,

$$
h\left(U^{\prime}\right) \leq h(U)+(\operatorname{deg} U) \log (N+1) \max \left(1, \frac{1}{2} D_{\ell}\right)+\log \left(\begin{array}{c}
\operatorname{deg} U+u_{\ell} \\
u_{\ell}
\end{array}\right) .
$$

Pour le degré, nous avons

$$
\operatorname{deg} Y_{\ell}^{\prime} \leq(\operatorname{deg} U)\left(\operatorname{deg} Y_{\ell}\right) \leq \Lambda^{\omega^{\prime} u \psi(u)} D_{\ell}
$$

TOME $133-2005-\mathrm{N}^{\mathrm{O}} 4$ 
et, en vertu de $\omega^{\prime} u \psi(u)+\psi(u)=\psi(u-1)$, cela entraîne bien les deuxième et troisième conditions dans la définition de $\left(Y_{1}^{\prime}, \ldots, Y_{m}^{\prime}\right) \in \mathcal{E}$ (la première étant quant à elle évidente par construction). Pour la hauteur, nous trouvons

$$
h\left(Y_{\ell}^{\prime}\right) \leq\left(\operatorname{deg} U^{\prime}\right) h\left(Y_{\ell}\right)+D_{\ell} h_{m}\left(U^{\prime}\right)
$$

où $h_{m}$ est la hauteur modifiée introduite dans [10] qui satisfait la majoration $h_{m}\left(U^{\prime}\right) \leq h\left(U^{\prime}\right)+\sqrt{N}$ d'après le lemme 5.2 de [11]. Ainsi

$$
\begin{aligned}
\sum_{i=1}^{m} a_{i} h\left(Y_{i}^{\prime}\right) & \leq D_{\ell} a_{\ell} h(U)+D_{\ell} a_{\ell} \Lambda^{\omega^{\prime} u \psi(u)} \log D_{\ell}(N+1)\left(u_{\ell}+1\right) \\
& +D_{\ell} a_{\ell} \sqrt{N}+\Lambda^{\omega^{\prime} u \psi(u)} \sum_{i=1}^{m} a_{i} h\left(Y_{i}\right) \\
& \leq \frac{1}{2} \Lambda^{\left(\omega^{\prime} u+\omega^{\prime}+1\right) \psi(u)+m+1-\omega^{\prime}}|a|\left(M t_{2}\right)^{m \operatorname{dim} X-u+1} \max (h(X), \delta)
\end{aligned}
$$

et ceci montre la dernière condition car $\left(\omega^{\prime} u+\omega^{\prime}+1\right) \psi(u)+m+1-\omega^{\prime} \leq 2 \psi(u-1)$ (cela vient par exemple de $m \leq u \psi(u)$ ).

Notons maintenant $\left(x_{0}^{(i)}, \ldots, x_{N}^{(i)}\right)$ un choix de coordonnées projectives de $\varphi_{i}\left(x_{i}\right)$ et $\left(z_{0}^{(i)}, \ldots, z_{N}^{(i)}\right)=M_{i}\left(x^{(i)}\right)$ qui est un choix possible pour $\chi_{i} \circ \varphi_{i}\left(x_{i}\right)$.

Corollaire 3.2. - Pour chaque indice $i$ avec $1 \leq i \leq m$, nous avons

1) le morphisme $\rho_{i}: Y_{i} \rightarrow \mathbb{P}_{\overline{\mathbb{Q}}}^{u_{i}}$ est étale au point $x_{i}$,

2) $z_{0}^{(i)} \neq 0$ et

3) pour tout $j$ avec $0 \leq j \leq N$,

$$
\frac{\partial^{b_{i, j}} Q_{j}^{(i)}}{\partial X_{u_{i}+1}^{b_{i, j}}}\left(1, \frac{z_{1}^{(i)}}{z_{0}^{(i)}}, \ldots, \frac{z_{u_{i}}^{(i)}}{z_{0}^{(i)}}, \frac{x_{j}^{(i)}}{z_{0}^{(i)}}\right) \neq 0 .
$$

Démonstration. - Il s'agit de montrer dans chaque cas que, si la condition est fausse, nous pouvons exhiber $U$ tel que $(i, U)$ satisfasse les conditions de la proposition. En notant $\Delta$ le discriminant du polynôme $P_{u_{i}+1}^{(i)}\left(V_{0}^{(i)}, \ldots, V_{u_{i}}^{(i)}, \cdot\right)$ et $R_{j}$ le résultant de $Q_{j}^{(i)}\left(V_{0}^{(i)}, \ldots, V_{u_{i}}^{(i)}, \cdot\right)$ et de sa dérivée d'ordre $b_{i, j}$, nous allons vérifier que $U=\Delta, U=V_{0}^{(i)}$ et $U=R_{j}$ conviennent.

En écrivant un résultant (et donc en particulier un discriminant) sous forme de déterminant, nous constatons que $\Delta$ et $R_{j}$ sont chacun égaux à celui d'une matrice carrée de taille au plus $2 D_{i}$; les coefficients de cette matrice sont des polynômes homogènes de $\overline{\mathbb{Q}}\left[V_{0}^{(i)}, \ldots, V_{u_{i}}^{(i)}\right]$ de degré au plus $D_{i}$; enfin, chaque coefficient de l'un de ces polynômes est le produit d'un élément de $B_{i}$ par un entier compris entre 1 et $D_{i}$ !.

Par suite $\operatorname{deg} \Delta$ et $\operatorname{deg} R_{j}$ sont majorés par $2 D_{i}^{2}$ tandis que $h(\Delta)$ et $h\left(R_{j}\right)$ sont au plus

$$
\log \left(2 D_{i}\right) !+2 D_{i} \log \left(\begin{array}{c}
D_{i} \\
u_{i}
\end{array}\right)+2 D_{i} \log D_{i} !+2 D_{i} h\left(B_{i}\right) \leq 6 N D_{i}^{3}+2 D_{i} h\left(B_{i}\right) .
$$

BULletin DE LA SOCIÉtÉ MATHÉMATIQUE DE FRANCE 
D'après le lemme 2.3, $h\left(B_{i}\right) \leq h\left(Y_{i}\right)+2 N^{2} D_{i}^{2}$ donc la borne ci-dessus devient

$$
\begin{aligned}
& 8 N^{2} D_{i}^{3}+\frac{\operatorname{deg} X}{a_{i}} \Lambda^{3 \psi(u)-1}|a|\left(M t_{2}\right)^{m \operatorname{dim} X-u} \max (h(X), \delta) \\
& \quad \leq \frac{1}{a_{i}} \Lambda^{3 \psi(u)-1}\left(\operatorname{deg} X+8 N^{2}(\operatorname{deg} X)^{3} \Lambda^{-2}\right)|a|\left(M t_{2}\right)^{m \operatorname{dim} X-u} \max (h(X), \delta) .
\end{aligned}
$$

Par suite, si $U$ vaut $\Delta, V_{0}^{(i)}$ ou $R_{j}$, les deux dernières conditions de la proposition sont vérifiées. Dans chaque cas, la définition d'un plongement adapté et celle de $P_{u_{i}+1}^{(i)}$ et $Q_{j}^{(i)}$ montrent qu'il en va de même de la seconde. Puisque la proposition affirme que les quatre conditions ne peuvent pas être réalisées simultanément, c'est donc la première qui est en défaut. Cela montre alors respectivement les trois assertions du présent corollaire qui est donc établi.

Puisque $z_{0}^{(i)}$ est non nul, nous pouvons, quitte à changer le choix de coordonnées fait plus haut, le supposer égal à 1 et c'est ce que nous ferons désormais.

Dans la mesure où le résultat vers lequel nous tendons consiste en une comparaison des hauteurs associées à $\mathcal{M}$ et $\mathcal{N}_{a}$, il est naturel de faire intervenir un faisceau écrit comme quotient (au sens du produit tensoriel) à partir de ces deux faisceaux. De manière précise, nous considérons les couples $(\varepsilon, d) \in \mathbb{Q} \times \mathbb{N}$ tels que $d \varepsilon \in \mathbb{Z}$ et associons à un tel couple le faisceau inversible sur $\mathcal{X}$

$$
\mathcal{Q}_{\varepsilon, d}=\mathcal{M}^{\otimes d} \otimes \mathcal{N}_{a}^{\otimes-d \varepsilon} .
$$

Le paramètre $d$ ainsi introduit est destiné à tendre vers l'infini : dans la suite les notations $o($.$) et O($.$) se réfèrent à cette limite.$

\section{Une petite section globale}

Dans toute cette partie, le point $x$ intervient uniquement à travers l'hypothèse sur le nombre d'intersection.

4.1. Dimension de l'espace des sections. - Dans ce paragraphe, de la donnée de $\left(\iota^{\prime}, j_{1}, j_{2}, \Sigma\right)$ nous ne retenons que l'existence d'une injection $j_{1}^{\prime}: \mathcal{M} \hookrightarrow \mathcal{N}_{a}^{\otimes t_{1}}$ sur $\mathcal{Y}$. Elle s'obtient en choisissant un élément de $\Sigma$ non nul sur $\mathcal{Y}$ et en composant l'injection $\mathcal{M} \hookrightarrow \mathcal{P}$ qui s'en déduit avec $j_{1}$.

Proposition 4.1. - Si l'on a $\varepsilon \leq(2 u \theta)^{-1}\left(t_{1} m\right)^{-u} \prod_{i=1}^{m} D_{i}^{-1-\omega}$, il existe $d_{0} \in \mathbb{N} \backslash\{0\}$ tel que si $d_{0}$ divise $d$,

$$
\operatorname{dim}_{\overline{\mathbb{Q}}} \Gamma\left(\mathcal{Y}, \mathcal{Q}_{\varepsilon, d}\right) \geq \frac{d^{u}}{4 \theta u !} \prod_{i=1}^{m} D_{i}^{-\omega} a_{i}^{u_{i}}+O\left(d^{u-1}\right) .
$$

TOME $133-2005-\mathrm{N}^{\mathrm{O}} 4$ 
Démonstration. - La théorie élémentaire de l'intersection montre que

$$
\left[\mathcal{N}_{a}\right]^{u} \cdot \mathcal{Y}=u ! \prod_{i=1}^{m} \frac{D_{i}}{u_{i} !} a_{i}^{u_{i}} \leq m^{u} \prod_{i=1}^{m} D_{i} a_{i}^{u_{i}}
$$

Considérons ensuite un sous-schéma $H$ de $\mathcal{Y}$ d'idéal isomorphe à $\mathcal{N}_{a}^{\otimes-1}$ et tel que la restriction de $j_{1}^{\prime}$ à $H$ est encore une injection : ceci est possible car $\mathcal{N}_{a}$ est engendré par ses sections globales sur $\mathcal{Y}$. Alors, pour tout faisceau $\mathcal{L}^{\prime}$ inversible sur $\mathcal{Y}$, la suite exacte

$$
0 \rightarrow \Gamma\left(\mathcal{Y}, \mathcal{L}^{\prime} \otimes \mathcal{N}_{a}^{\otimes-1}\right) \longrightarrow \Gamma\left(\mathcal{Y}, \mathcal{L}^{\prime}\right) \longrightarrow \Gamma\left(H, \mathcal{L}^{\prime}\right)
$$

permet d'écrire $\operatorname{dim} \Gamma\left(\mathcal{Y}, \mathcal{L}^{\prime} \otimes \mathcal{N}_{a}^{\otimes-1}\right) \geq \operatorname{dim} \Gamma\left(\mathcal{Y}, \mathcal{L}^{\prime}\right)-\operatorname{dim} \Gamma\left(H, \mathcal{L}^{\prime}\right)$. Par suite, en itérant cette formule, nous avons pour tout $d_{0}$ tel que $d_{0}$ divise $d$,

$$
\operatorname{dim}_{\overline{\mathbb{Q}}} \Gamma\left(\mathcal{Y}, \mathcal{Q}_{\varepsilon, d}\right) \geq \operatorname{dim}_{\overline{\mathbb{Q}}} \Gamma\left(\mathcal{Y}, \mathcal{Q}_{-1 / d_{0}, d}\right)-\sum_{j=-d / d_{0}}^{d \varepsilon-1} \operatorname{dim}_{\overline{\mathbb{Q}}} \Gamma\left(H, \mathcal{N}_{a}^{\otimes t_{1} d-j}\right) .
$$

En faisant usage de $j_{1}^{\prime}$, il vient $\operatorname{dim} \Gamma\left(H, \mathcal{Q}_{j / d, d}\right) \leq \operatorname{dim} \Gamma\left(H, \mathcal{N}_{a}^{\otimes t_{1} d-j}\right)$ puis

$$
\begin{aligned}
& \sum_{j=-d / d_{0}}^{d \varepsilon-1} \operatorname{dim}_{\overline{\mathbb{Q}}} \Gamma\left(H, \mathcal{Q}_{j / d, d}\right) \\
& \quad \leq \sum_{j=-d / d_{0}}^{d \varepsilon-1} \frac{\left(t_{1} d-j\right)^{u-1}}{(u-1) !}\left[\mathcal{N}_{a}\right]^{u-1} \cdot H+O\left(d^{u-1}\right) \\
& \quad \leq \sum_{j=-d / d_{0}}^{d \varepsilon-1} \frac{\left(t_{1} d-j\right)^{u-1}}{(u-1) !}\left[\mathcal{N}_{a}\right]^{u} \cdot \mathcal{Y}+O\left(d^{u-1}\right) \\
& \quad \leq u m^{u}\left(t_{1}+\frac{1}{d_{0}}\right)^{u-1} \frac{d^{u}}{u !}\left(\prod_{i=1}^{m} D_{i} a_{i}^{u_{i}}\right) \sum_{j=-d / d_{0}}^{d \varepsilon-1} \frac{1}{d}+O\left(d^{u-1}\right) \\
& \quad \leq\left(1+\frac{1}{\varepsilon d_{0}}\right)^{u} \frac{d^{u}}{2 \theta u !} \prod_{i=1}^{m} D_{i}^{-\omega} a_{i}^{u_{i}}+O\left(d^{u-1}\right)
\end{aligned}
$$

en utilisant la majoration de $\varepsilon$ (qui entraîne en particulier $\varepsilon \leq 1 \leq t_{1}$ ). D'autre part

$$
\begin{aligned}
\operatorname{dim}_{\overline{\mathbb{Q}}} \Gamma\left(\mathcal{Y}, \mathcal{Q}_{-1 / d_{0}, d}\right) & =\frac{\left(d / d_{0}\right)^{u}}{u !}\left[\mathcal{M}^{\otimes d_{0}} \otimes \mathcal{N}_{a}\right]^{u} \cdot \mathcal{Y}+O\left(d^{u-1}\right) \\
& =\frac{d^{u}}{u !}\left([\mathcal{M}]^{u} \cdot \mathcal{Y}+P_{1}\left(\frac{1}{d_{0}}\right)\right)+O\left(d^{u-1}\right) \\
& \geq \frac{d^{u}}{\theta u !} \prod_{i=1}^{m} D_{i}^{-\omega} a_{i}^{u_{i}}\left(1+P_{2}\left(\frac{1}{d_{0}}\right)\right)+O\left(d^{u-1}\right)
\end{aligned}
$$

BULlETIN DE LA SOCIÉTÉ MATHÉMATIQUE DE FRANCE 
d'après l'hypothèse sur $[\mathcal{M}]^{u} \cdot \mathcal{Y}$; ici, $P_{1}$ et $P_{2}$ désignent des éléments de $T \mathbb{Q}[T]$ indépendants de $d$ et $d_{0}$. En combinant les deux estimations, il reste à choisir $d_{0}$ de sorte que

$$
1+P_{2}\left(\frac{1}{d_{0}}\right)-\frac{1}{2}\left(1+\frac{1}{\varepsilon d_{0}}\right)^{u} \geq \frac{1}{4}
$$

et cela termine la preuve.

Nous fixons dorénavant

$$
\varepsilon=\frac{1}{2 u \theta} \frac{1}{\left(t_{1} m\right)^{u}} \prod_{i=1}^{m} D_{i}^{-1-\omega}
$$

et un entier $d_{0}$ donné par la proposition. Nous considérons toujours que $d$ est tel que $d_{0}$ divise $d$.

4.2. Contrôle de hauteur. — Afin d'exploiter un lemme de Siegel, nous allons écrire $\mathcal{Q}_{\varepsilon, d}$ comme le noyau d'un morphisme de faisceaux bien contrôlé. Soit d'abord $\mathcal{N}=\mathcal{M}^{\otimes-d} \otimes \mathcal{P}^{\otimes d} \otimes \mathcal{N}_{a}^{\otimes \varepsilon d}$. Nous considérons la famille $\Sigma^{\prime}$ de sections globales de $\mathcal{N}$ de la forme

$$
\sigma^{\otimes d} \otimes \pi^{*} \bigotimes_{i=1}^{m} W_{\ell_{i}}^{(i) d \varepsilon a_{i}}
$$

où $\sigma \in \Sigma$ et $0 \leq \ell_{i} \leq N$ pour $1 \leq i \leq m$. Cette famille engendre $\mathcal{N}$ et a pour cardinal $M(N+1)^{m}$. Elle donne donc naissance à une suite exacte (quelquefois appelée dans ce contexte complexe de Faltings)

$$
0 \rightarrow \mathcal{Q}_{\varepsilon, d} \stackrel{\Phi}{\longrightarrow} \bigoplus_{\sigma \in \Sigma^{\prime}} \mathcal{Q}_{\varepsilon, d} \otimes \mathcal{N}-\stackrel{\Psi^{\prime}}{\longrightarrow} \underset{\sigma_{1}, \sigma_{2} \in \Sigma^{\prime}}{\bigoplus} \mathcal{Q}_{\varepsilon, d} \otimes \mathcal{N}^{\otimes 2}
$$

avec

$$
\Phi(s)=(s \otimes \sigma)_{\sigma \in \Sigma^{\prime}} \quad \text { et } \quad \Psi^{\prime}\left(\left(s_{\sigma}\right)_{\sigma \in \Sigma^{\prime}}\right)=\left(s_{\sigma_{1}} \otimes \sigma_{2}-s_{\sigma_{2}} \otimes \sigma_{1}\right)_{\sigma_{1}, \sigma_{2} \in \Sigma^{\prime}} .
$$

Grâce à $j_{1}$ et $j_{2}$ nous avons une injection

$$
\mathcal{Q}_{\varepsilon, d} \otimes \mathcal{N}^{\otimes 2} \simeq \mathcal{P}^{\otimes 2 d} \otimes \mathcal{M}^{\otimes-d} \otimes \mathcal{N}_{a}^{\otimes \varepsilon d} \longleftrightarrow \mathcal{N}_{a}^{\otimes d\left(t_{1}+t_{2}+\varepsilon\right)} .
$$

En définissant $\Psi$ comme la composée de $\Psi^{\prime}$ avec celle-ci, nous aboutissons à la suite exacte

$$
0 \rightarrow \mathcal{Q}_{\varepsilon, d} \stackrel{\Phi}{\longrightarrow} \bigoplus_{\Sigma^{\prime}} \mathcal{P}^{\otimes d} \stackrel{\Psi}{\longrightarrow} \bigoplus_{\left(\Sigma^{\prime}\right)^{2}} \mathcal{N}_{a}^{\otimes d\left(t_{1}+t_{2}+\varepsilon\right)} .
$$

Nous supposons $d$ assez grand pour que toutes les sections globales de $\mathcal{P}^{\otimes d}$ sur $\mathcal{Y}$ proviennent de $\mathbb{P}_{\overline{\mathbb{Q}}}^{N^{\prime}}$ et nous fixons aussi une fois pour toute une famille de monômes en les coordonnées de $\mathbb{P} N_{\overline{\mathbb{Q}}}^{N^{\prime}}$ de degré $d$ (engendrant un espace $E$ ) dont les images forment une base de $\Gamma\left(\mathcal{Y}, \mathcal{P}^{\otimes d}\right)$.

TOME $133-2005-\mathrm{N}^{\mathrm{O}} 4$ 
Nous dirons que $s \in \Gamma\left(\mathcal{Y}, \mathcal{Q}_{\varepsilon, d}\right)$ est représentée par une famille de polynômes $F_{\sigma} \in E\left(\sigma \in \Sigma^{\prime}\right)$ si l'image de $\left(F_{\sigma}\right)_{\sigma}$ coïncide avec $\Phi(s)$.

Proposition 4.2. - Il existe une section $s \in \Gamma\left(\mathcal{Y}, \mathcal{Q}_{\varepsilon, d}\right)$ non nulle représentée par une famille $\mathcal{F}=\left(F_{\sigma}\right)_{\sigma}$ de hauteur majorée par

$d M(N+1)^{m} 4\left(t_{1}+t_{2}+\varepsilon\right) \theta\left(t_{1} m\right)^{u}\left(\prod_{i=1}^{m} D_{i}^{1+\omega}\right) \sum_{i=1}^{m} a_{i}\left(h\left(B_{i}\right)+\delta+\log \left(2 u_{i}+2\right)\right)+o(d)$.

Démonstration. - La méthode consiste à déduire du morphisme

$$
F=\bigoplus_{\Sigma^{\prime}} E \longrightarrow \bigoplus_{\left(\Sigma^{\prime}\right)^{2}} \Gamma\left(\mathcal{Y}, \mathcal{N}_{a}^{\otimes d\left(t_{1}+t_{2}+\varepsilon\right)}\right)
$$

(donné par $\Psi$ ) une famille $L_{1}, \ldots, L_{b}$ de formes linéaires sur $F$ définissant son noyau, isomorphe à $\Gamma\left(\mathcal{Y}, \mathcal{Q}_{\varepsilon, d}\right)$. D'après le lemme de Siegel (voir lemme 2.6), nous aurons notre résultat avec

$$
h(\mathcal{F}) \leq \frac{\operatorname{dim} F}{\operatorname{dim} \Gamma\left(\mathcal{Y}, \mathcal{Q}_{\varepsilon, d}\right)}\left(h\left(L_{1}, \ldots, L_{b}\right)+\log \operatorname{dim} F+c_{S}\left(\mathbb{Q}\left(L_{1}, \ldots, L_{b}\right)\right)\right)
$$

où $\mathbb{Q}\left(L_{1}, \ldots, L_{b}\right)$ est le corps engendré par les coefficients des $L_{j}$, ces derniers (qui servent aussi à définir la hauteur $h\left(L_{1}, \ldots, L_{b}\right)$ ) étant pris dans la base duale de celle choisie pour $F$.

Grâce à l'injection $\mathcal{P}^{\otimes d} \hookrightarrow \mathcal{N}_{a}^{\otimes d t_{1}}$, nous avons

$$
\operatorname{dim} E \leq \prod_{i=1}^{m} \frac{D_{i}}{u_{i} !}\left(d t_{1} a_{i}\right)^{u_{i}} \leq\left(t_{1} m\right)^{u} \frac{d^{u}}{u !} \prod_{i=1}^{m} D_{i} a_{i}^{u_{i}} .
$$

Par conséquent $\log \operatorname{dim} F=o(d)$ et, d'après la proposition 4.1,

$$
\frac{\operatorname{dim} F}{\operatorname{dim} \Gamma\left(\mathcal{Y}, \mathcal{Q}_{\varepsilon, d}\right)} \leq 4 \theta M(N+1)^{m}\left(t_{1} m\right)^{u} \prod_{i=1}^{m} D_{i}^{1+\omega}+o(1) .
$$

Le résultat sera donc acquis si nous savons choisir les $L_{j}$ à coefficients dans un corps indépendant de $d\left(\right.$ de sorte que $\left.c_{S}\left(\mathbb{Q}\left(L_{1}, \ldots, L_{b}\right)\right)=o(d)\right)$ et tels que

$$
h\left(L_{1}, \ldots, L_{b}\right) \leq d\left(t_{1}+t_{2}+\varepsilon\right) \sum_{i=1}^{m} a_{i}\left(h\left(B_{i}\right)+\delta+\log \left(2 u_{i}+2\right)\right)+o(d) .
$$

Une façon naïve de choisir des $L_{j}$ serait de fixer une base de $\Gamma\left(\mathcal{Y}, \mathcal{N}_{a}^{\otimes d\left(t_{1}+t_{2}+\varepsilon\right)}\right)$ et d'y décomposer $\Psi(\mathcal{F})$. Cependant, pour pouvoir estimer la hauteur des coefficients qui apparaissent, il faudrait connaitre assez explicitement une telle base. En pratique, nous procédons un peu différemment. Nous notons

$$
\Theta: \overline{\mathbb{Q}}[W] \simeq \bigoplus_{\gamma} \Gamma\left(\left(\mathbb{P}_{\overline{\mathbb{Q}}}^{N}\right)^{m}, \mathcal{O}(\gamma)\right) \longrightarrow \bigoplus_{\gamma} \Gamma\left(\mathcal{Y}, \mathcal{N}_{\gamma}\right)
$$

où $\gamma$ parcourt $\mathbb{N}^{m}$. Nous voyons les $V_{j}^{(i)}$ comme éléments de $\overline{\mathbb{Q}}[W]$ (par $\left.\left(V^{(i)}\right)=M_{i}\left(W^{(i)}\right)\right)$ et les choix de plongements adaptés montrent que $\Theta$ se BULLETIN DE LA SOCIÉtÉ MATHÉMATIQUE DE FRANCE 
restreint injectivement à l'espace $\mathcal{R}$ des polynômes $R$ en $V$ avec $\operatorname{deg}_{V_{j}^{(i)}} R=0$ si $u_{i}+2 \leq j \leq n$ et $\operatorname{deg}_{V_{u_{i}+1}^{(i)}} R<D_{i}$. Nous connaissons donc une base $\operatorname{de} \Theta(\mathcal{R})$ et nous allons ramener les calculs dans cet espace.

Pour cela, puisque (le plongement étant adapté) $v_{u_{i}+1}^{(i)}$ engendre $K\left(Y_{i}\right)$ sur $K\left(v_{1}^{(i)}, \ldots, v_{u_{i}}^{(i)}\right)$, il existe un élément $R_{Y}$ de $\mathcal{R}$ non nul tel que l'on puisse écrire pour tout élément de l'ensemble fini $\mathcal{K}=\left\{k \in \mathbb{N}^{(N+1) m} ; k_{i, j}<D_{i}\right\}$

$$
\Theta\left(R_{Y} W^{k}\right)=\Theta\left(S_{k}\right)
$$

où $S_{k} \in \mathcal{R}$. Si nous regardons maintenant une composante de $\Psi(\mathcal{F})$ dans $\Gamma\left(\mathcal{Y}, \mathcal{N}_{a}^{\otimes d\left(t_{1}+t_{2}+\varepsilon\right)}\right)$ (c'est-à-dire pour un élément de $\Sigma^{\prime}$ fixé), nous constatons avec les définitions que le terme en facteur d'un coefficient donné de $\mathcal{F}$ vaut (s'il est non nul)

$$
\pm \Theta\left(\mathfrak{m} P_{j}^{d}\right)
$$

où $1 \leq j \leq M$ et $\mathfrak{m}$ est un monôme en $W$ unitaire de multidegré $d\left(t_{1}+\varepsilon\right) a$. Si nous appliquons alors le lemme 2.5 à la famille des polynômes $\mathfrak{m} P_{j}^{d}$, nous trouvons des polynômes $Q_{k, j, \mathfrak{m}}$ ne faisant intervenir que les $V_{j}^{(i)}$ avec $j \leq u_{i}$, définis sur un corps de nombres indépendant de $d$, vérifiant

$h\left(\left(Q_{k, j, \mathfrak{m}}\right)_{k, j, \mathfrak{m}}\right) \leq h\left(P_{1}^{d}, \ldots, P_{M}^{d}\right)+\sum_{i=1}^{m} d\left(t_{1}+t_{2}+\varepsilon\right) a_{i}\left(h\left(B_{i}\right)+\log \left(2 u_{i}+2\right)\right)+o(d)$

et tels que

$$
\Theta\left(\mathfrak{m} P_{j}^{d}\right)=\Theta\left(\sum_{k \in \mathcal{K}} Q_{k, j, \mathfrak{m}} W^{k}\right) .
$$

Finalement, puisque $\Psi(\mathcal{F})=0$ équivaut à $\Psi(\mathcal{F}) \otimes \Theta\left(R_{Y}\right)=0$, nous pouvons réécrire la condition sur $\mathcal{F}$ sous forme d'équations où le terme en facteur d'un coefficient donné de $\mathcal{F}$ est

$$
\pm \Theta\left(\sum_{k \in \mathcal{K}} Q_{k, j, \mathfrak{m}} S_{k}\right)
$$

Les polynômes apparaissant ainsi appartiennent à $\mathcal{R}$ donc les formes linéaires cherchées s'obtiennent grâce à la base de $\mathcal{R}$ formée des monômes en $V$ contenus dans cet espace. Les coefficients ainsi fabriqués engendrent bien un corps de nombres indépendant de $d$ et la hauteur de leur famille, puisque $S_{k}$ est indépendant de $d$ tout comme le cardinal de $\mathcal{K}$, est majorée par

$$
h\left(\left(Q_{k, j, \mathfrak{m}}\right)_{k, j, \mathfrak{m}}\right)+o(d) \leq d \delta|a|+\sum_{i=1}^{m} d\left(t_{1}+t_{2}+\varepsilon\right) a_{i}\left(h\left(B_{i}\right)+\log \left(2 u_{i}+2\right)\right)+o(d)
$$

donc par la quantité annoncée et cela clôt la démonstration.

TOME $133-2005-\mathrm{N}^{\mathrm{O}} 4$ 


\section{Ordre d'annulation}

Si la section $s$ ne s'annulait pas en $x$, nous pourrions aisément écrire une minoration de la hauteur associée à $\mathcal{Q}_{\varepsilon, d}$ valable en $x$ et la conclusion du théorème 1.2 en découlerait. Si elle s'annule en $x$ mais avec un ordre suffisamment petit, il est possible de raisonner de la même façon et, quitte à faire intervenir des dérivations, d'obtenir la même conclusion. C'est ce que nous faisons cidessous : après avoir déduit du lemme 2.7 une estimation pour les dérivées de certains monômes, nous introduisons la notion précise d'indice qui contrôle l'annulation de $s$ et montrons le lien avec les hauteurs. Finalement, nous traitons le cas où l'indice est grand à l'aide du théorème du produit de Faltings : grâce à l'hypothèse $a_{i} / a_{i+1} \leq c_{2}$, l'annulation doit se faire le long d'un sous-produit de $Y=Y_{1} \times \cdots \times Y_{m}$ et les estimations font que celui-ci contredit la minimalité de $Y$.

\subsection{Estimation locale des dérivées. — Le morphisme}

$$
\rho: \mathcal{Y} \longrightarrow \mathbb{P}_{\overline{\mathbb{Q}}}^{u_{1}} \times \cdots \times \mathbb{P}_{\overline{\mathbb{Q}}}^{u_{m}}
$$

déduit des $\rho_{i}$ est étale au point $x$. Nous pouvons donc trouver un voisinage ouvert de $x$ dans $\mathcal{Y}$ au-dessus duquel $\Omega_{\mathcal{Y} / \overline{\mathbb{Q}}}$ ait pour base la famille $\left(d v_{j}^{(i)}\right)_{i, j}$ avec $1 \leq i \leq m$ et $1 \leq j \leq u_{i}$. Notons $\partial_{v_{j}^{(i)}}$ les dérivations correspondantes $\left(\partial_{v_{j}^{(i)}}\left(v_{j^{\prime}}^{\left(i^{\prime}\right)}\right)=\delta_{i, i^{\prime}} \delta_{j, j^{\prime}}\right)$ et posons pour $\ell \in \mathbb{N}^{u_{i}}$

$$
\partial^{i, \ell}=\prod_{j=1}^{u_{i}} \frac{1}{\ell_{j} !}\left(\partial_{v_{j}^{(i)}}\right)^{\ell_{j}} .
$$

Soit $K$ un corps de nombres contenant les coordonnées $x_{j}^{(i)}$ et les familles $B_{i}$. Par application du lemme 2.7, nous obtenons le

Corollaire 5.1. - Si $v$ est une place de $K, 1 \leq i \leq m, 0 \leq j \leq N$, $\ell \in(\mathbb{N} \backslash\{0\})^{u_{i}}$ et $\eta \in\{-1,1\}$, on a

$$
\begin{aligned}
& \left|\partial^{i, \ell}\left(w_{j}^{(i) \eta}\right)(x)\right|_{v} \\
& \quad \leq\left|x_{j}^{(i)}\right|_{v} f_{1}\left(u_{i}, D_{i}\right)^{\varepsilon_{v}|\ell|}\left(\frac{\left|Q_{j}^{(i)}\left(1, X_{1}+z_{1}^{(i)}, \ldots, X_{u_{i}}+z_{u_{i}}^{(i)}, x_{j}^{(i)} Y\right)\right|_{v}}{\left|\frac{x_{j}^{(i)^{b_{i, j}}}}{b_{i, j} !} \frac{\partial^{b_{i, j}} Q_{j}^{(i)}}{\partial X_{u_{i}+1}^{b_{i, j}}}\left(1, z_{1}^{(i)}, \ldots, z_{u_{i}}^{(i)}, x_{j}^{(i)}\right)\right|_{v}}\right)^{\frac{2|\ell|-1}{b_{i, j}}} .
\end{aligned}
$$

Démonstration. - Pour $\eta=1$, c'est une application directe du lemme avec $X_{k}=v_{k}^{(i)}-z_{k}^{(i)}\left(1 \leq k \leq u_{i}\right), y=w_{j}^{(i)}$ et $a=x_{j}^{(i)}$ ( $\varphi$ étant l'évaluation en $x)$. Pour $\eta=-1$, il faut considérer le polynôme réciproque de $Q_{j}^{(i)}$ en $X_{u_{i}+1}$ et on a le résultat en reliant sa dérivée à celle de $Q_{j}^{(i)}$. 
Ceci nous amène à poser (pour un choix quelconque de racines)

$$
c_{i}=\prod_{k=0}^{N}\left(\frac{x_{k}^{(i)} b_{i, k}}{b_{i, k} !} \frac{\partial^{b_{i, k}} Q_{k}^{(i)}}{\partial X_{u_{i}+1}^{b_{i, k}}}\left(1, z_{1}^{(i)}, \ldots, z_{u_{i}}^{(i)}, x_{k}^{(i)}\right)\right)^{1 / b_{i, k}} \quad(1 \leq i \leq m)
$$

et nous supposons que $K$ contient ces éléments. Nous pouvons alors écrire une majoration plus commode des dérivées.

LEMme 5.2. - Sous les hypothèses du corollaire précédent, on a

$$
\left|c_{i}^{2|\ell|} \partial^{i, \ell}\left(w_{j}^{(i)^{\eta}}\right)(x)\right|_{v} \leq\left|x_{j}^{(i)^{\eta}}\right|_{v} f_{2}\left(u_{i}, D_{i}\right)^{\varepsilon_{v}|\ell|}\left(\left|B_{i}\right|_{v} \max _{0 \leq k \leq N}\left|x_{k}^{(i)}\right|_{v}^{D_{i}}\right)^{2(N+1)|\ell|}
$$

où

$$
f_{2}(u, D)=f_{1}(u, D)\left(\begin{array}{c}
D+u \\
u
\end{array}\right)^{2(N+1)}(2(N+1) D)^{2(N+1) D} .
$$

Démonstration. - C'est évident si $|\ell|=0$; sinon nous appliquons le corollaire. Le facteur de $c_{i}$ correspondant à $k=j$ est utilisé pour supprimer le dénominateur apparaissant dans le corollaire. Il reste un produit de facteurs à majorer : tout d'abord en notant ici pour $0 \leq k \leq N$

$$
A_{k}=2^{\varepsilon_{v} D_{i}}\left(\begin{array}{c}
D_{i}+u_{i} \\
u_{i}
\end{array}\right)^{\varepsilon_{v}}\left|B_{i}\right|_{v} \max \left(1,\left|z_{1}^{(i)}\right|_{v}, \ldots,\left|z_{u_{i}}^{(i)}\right|_{v},\left|x_{k}^{(i)}\right|_{v}\right)^{D_{i}}
$$

il vient

$$
\left|Q_{j}^{(i)}\left(1, X_{1}+z_{1}^{(i)}, \ldots, X_{u_{i}}+z_{u_{i}}^{(i)}, x_{j}^{(i)} Y\right)\right|_{v} \leq A_{j}
$$

De manière analogue,

$$
\begin{aligned}
& \left|\frac{x_{k}^{(i)} b_{i, k}}{b_{i, k} !} \frac{\partial^{b_{i, k}} Q_{k}^{(i)}}{\partial X_{u_{i}+1}^{b_{i, k}}}\left(1, z_{1}^{(i)}, \ldots, z_{u_{i}}^{(i)}, x_{k}^{(i)}\right)\right|_{v} \\
& \quad \leq\left(\begin{array}{c}
D_{i}+u_{i}+1 \\
u_{i}+b_{i, k}+1
\end{array}\right)^{\varepsilon_{v}}\left|B_{i}\right|_{v} \max \left(1,\left|z_{1}^{(i)}\right|_{v}, \ldots,\left|z_{u_{i}}^{(i)}\right|_{v},\left|x_{k}^{(i)}\right|_{v}\right)^{D_{i}}
\end{aligned}
$$

puis $\left(\begin{array}{c}D_{i}+u_{i}+1 \\ u_{i}+b_{i, k}+1\end{array}\right) \leq\left(\begin{array}{c}D_{i}+u_{i}+1 \\ u_{i}+2\end{array}\right)^{b_{i, k}}$. En utilisant encore $\left(\begin{array}{c}D_{i}+u_{i}+1 \\ u_{i}+2\end{array}\right) \leq 2^{D_{i}}\left(\begin{array}{c}D_{i}+u_{i} \\ u_{i}\end{array}\right)$, nous trouvons

$$
\left|\frac{x_{k}^{(i)} b_{i, k}}{b_{i, k} !} \frac{\partial^{b_{i, k}} Q_{k}^{(i)}}{\partial X_{u_{i}+1}^{b_{i, k}}}\left(1, z_{1}^{(i)}, \ldots, z_{u_{i}}^{(i)}, x_{k}^{(i)}\right)\right|_{v}^{1 / b_{i, k}} \leq A_{k} .
$$

Puisque $2|\ell|-1>0$, la quantité de l'énoncé est au plus

$$
\left|x_{j}^{(i)^{\eta}}\right|_{v} f_{1}\left(u_{i}, D_{i}\right)^{\varepsilon_{v}|\ell|} \prod_{k=0}^{N} A_{k}^{2|\ell|}
$$

et l'on conclut par $\left|z_{1}^{(i)}\right|_{v}, \ldots,\left|z_{u_{i}}^{(i)}\right|_{v} \leq(N+1) D_{i} \max _{0 \leq k \leq N}\left|x_{k}^{(i)}\right|_{v}$ puisque $\left(z^{(i)}\right)=M_{i}\left(x^{(i)}\right)$.

TOME $133-2005-\mathrm{N}^{\mathrm{O}} 4$ 
5.2. Indice. - Nous notons $\sigma$ l'indice au point $x$ et relativement aux poids $d t_{1} a$ de la section $s \in \Gamma\left(\mathcal{Y}, \mathcal{Q}_{\varepsilon, d}\right)$ dont l'existence est assurée par la proposition 4.2. Par définition, cela signifie qu'il existe $\kappa \in \prod_{i=1}^{m} \mathbb{N}^{u_{i}}$ tel que $\sum_{i=1}^{m}\left|\kappa_{i}\right| / d t_{1} a_{i}=\sigma$ et ayant la propriété suivante : si $s$ s'écrit $\alpha s^{\prime}$ au voisinage de $x$ où $\alpha$ est une section de $\mathcal{O}_{\mathcal{Y}}$ et $s^{\prime}$ une section de $\mathcal{Q}_{\varepsilon, d}$ non nulle en $x$, alors on a, si $\sum_{i=1}^{m}\left|\kappa_{i}^{\prime}\right| / d t_{1} a_{i}<\sigma$,

$$
\left(\prod_{i=1}^{m} \partial^{i, \kappa_{i}}\right)(\alpha)(x) \neq 0 \quad \text { et } \quad\left(\prod_{i=1}^{m} \partial^{i, \kappa_{i}^{\prime}}\right)(\alpha)(x)=0 .
$$

L'objet de ce paragraphe consiste à montrer que si la section $s$ ne s'annule pas trop en $x$, au sens de l'indice $\sigma$ ainsi défini, alors la hauteur associée à $\mathcal{Q}_{\varepsilon, d}$ est minorée en $x$. De manière précise, nous avons :

Proposition 5.3. - Si $\sigma \leq\left(4 t_{1}(N+1) \max _{i} D_{i}\right)^{-1} \varepsilon$, alors

$$
\sum_{i=1}^{m} a_{i} h_{\mathcal{L}}\left(x_{i}\right) \leq c_{1} h_{\mathcal{M}}(x)
$$

Nous partons de la quantité suivante (qui est bien une hauteur associée à $\left.\mathcal{Q}_{\varepsilon, d}\right)$

$$
\phi=d h\left(\iota^{\prime}(x)\right)-d h(\Sigma(x))-d \varepsilon \sum_{i=1}^{m} a_{i} h_{\mathcal{L}}\left(x_{i}\right) .
$$

Nous choisissons des coordonnées $\xi_{0}, \ldots, \xi_{N^{\prime}}$ pour $\iota^{\prime}(x)$ et $\xi_{1}^{\prime}, \ldots, \xi_{M}^{\prime}$ pour $\Sigma(x)$. Nous fixons un corps de nombres $K$ comme dans le paragraphe précédent contenant en plus ces coordonnées et les coefficients de $\mathcal{F}$. Alors on a

$$
\phi=\sum_{v} \frac{\left[K_{v}: \mathbb{Q} v\right]}{[K: \mathbb{Q}]} \log \left(\max _{0 \leq j \leq N^{\prime}}\left|\xi_{j}\right|_{v}^{d} \cdot \min _{1 \leq k \leq M}\left|\xi_{k}^{\prime}\right|_{v}^{-d} \cdot \prod_{i=1}^{m} \min _{0 \leq \ell_{i} \leq N}\left|x_{\ell_{i}}^{(i)}\right|_{v}^{-d \varepsilon a_{i}}\right)
$$

où la somme porte sur toutes les places de $K$. Pour chaque telle place, choisissons $j_{v}, k_{v}$ et $\left(\ell_{v, i}\right)_{i}$ réalisant respectivement le maximum et les minima apparaissant; ainsi

$$
\phi=\sum_{v} \frac{\left[K_{v}: \mathbb{Q}_{v}\right]}{[K: \mathbb{Q}]} \log \left|\xi_{j_{v}}^{d} \xi_{k_{v}}^{\prime-d} \prod_{i=1}^{m} x_{\ell_{v, i}}^{(i)}{ }^{-d \varepsilon a_{i}}\right|_{v} .
$$

Si $\left(\Xi_{j}\right)$ et $\left(\Xi_{k}^{\prime}\right)$ sont les coordonnées respectives de $\mathbb{P}_{\overline{\mathbb{Q}}}^{N^{\prime}}$ et $\mathbb{P}_{\overline{\mathbb{Q}}}^{M-1}$ et si $f: \mathcal{Y} \rightarrow$ $\mathbb{P}_{\overline{\mathbb{Q}}}^{M-1}$ est donné par $\Sigma$, alors la quantité qui apparaît est reliée à la section

$$
s_{v}=\iota^{\prime *} \Xi_{j_{v}}^{\otimes d} \otimes f^{*} \Xi_{k_{v}}^{\prime \otimes-d} \otimes \bigotimes_{i=1}^{m} \pi^{*} p_{i}^{*} W_{\ell_{v, i}}^{\otimes-d \varepsilon a_{i}}
$$

BULletin DE LA SOCiÉtÉ MATHÉMATIQUE DE FRANCE 
de $\mathcal{Q}_{\varepsilon, d}$ sur un voisinage de $x$. Parallèlement, par définition de $\mathcal{F}$, nous avons

$$
s=\iota^{\prime *} F_{k_{v}, \ell_{v}}(\Xi) \otimes f^{*} \Xi_{k_{v}}^{\prime \otimes-d} \otimes \bigotimes_{i=1}^{m} \pi^{*} p_{i}^{*} W_{\ell_{v, i}}^{\otimes-d \varepsilon a_{i}}
$$

également sur un voisinage de $x$ (si l'on décrit $\Sigma^{\prime}$ par les couples $(k, \ell)$ ). Par conséquent, si nous choisissons une section $s^{\prime}$ non nulle au voisinage de $x$ (par exemple l'une des $s_{v}$ ) et écrivons $s_{v}=\alpha_{v} s^{\prime}$ et $s=\alpha s^{\prime}$, nous avons

$$
\phi=\sum_{v} \frac{\left[K_{v}: \mathbb{Q}_{v}\right]}{[K: \mathbb{Q}]} \log \left|\alpha_{v}(x)\right|_{v} \quad \text { et } \quad \alpha=\alpha_{v} F_{k_{v}, \ell_{v}}\left(\Xi / \Xi_{j_{v}}\right)
$$

pour chaque $v$, où $\Xi_{j} / \Xi_{j}$ est vu dans le corps des fonctions de $\mathcal{Y}$. Enfin, en notant

$$
D=\prod_{i=1}^{m} \partial^{i, \kappa_{i}}
$$

l'opérateur dont la définition de $\sigma$ assure l'existence, il vient

$$
(D \alpha)(x)=\alpha_{v}(x) \cdot D\left(F_{k_{v}, \ell_{v}}\left(\Xi / \Xi_{j_{v}}\right)\right)(x)
$$

et donc, par la formule du produit pour $(D \alpha)(x) \neq 0$,

$$
\phi=-\sum_{v} \frac{\left[K_{v}: \mathbb{Q}_{v}\right]}{[K: \mathbb{Q}]} \log \left|D\left(F_{k_{v}, \ell_{v}}\left(\Xi / \Xi_{j_{v}}\right)\right)(x)\right|_{v} .
$$

Il est à présent possible de minorer $\phi$. Dans un premier temps,

$$
-\phi \leq h(\mathcal{F})+\log \operatorname{dim} E+\sum_{v} \frac{\left[K_{v}: \mathbb{Q} v\right]}{[K: \mathbb{Q}]} \log \max _{\mathfrak{m}}\left|D\left(\mathfrak{m}\left(\Xi / \Xi_{j_{v}}\right)\right)(x)\right|_{v}
$$

où $\mathfrak{m}$ parcourt les éléments de la base de $E$ que l'on a fixée, qui sont des monômes de degré $d$. Grâce à l'hypothèse faite sur $j_{1}$, un tel monôme s'écrit

$$
\mathfrak{m}\left(\Xi / \Xi_{j_{v}}\right)=\prod_{i=1}^{m} \prod_{\lambda=1}^{2 d t_{1} a_{i}}\left(w_{\nu_{\lambda, i}}^{(i)}\right)^{\eta_{\lambda, i}}
$$

avec $0 \leq \nu_{\lambda, i} \leq N$ et $\eta_{\lambda, i} \in\{-1,1\}$ pour tous $i, \lambda$. Par la formule de Leibniz,

$$
D\left(\mathfrak{m}\left(\Xi / \Xi_{j_{v}}\right)\right)=\prod_{i=1}^{m} \sum_{\left(\ell_{\lambda}\right)} \prod_{\lambda=1}^{2 d t_{1} a_{i}} \partial^{i, \ell_{\lambda}}\left(\left(w_{\nu_{\lambda, i}}^{(i)}\right)^{\eta_{\lambda, i}}\right)
$$

où la somme est sur toutes les familles $\left(\ell_{\lambda}\right)$ d'éléments de $\mathbb{N}^{u_{i}}$ qui vérifient $\sum_{\lambda=1}^{2 d t_{1} a_{i}} \ell_{\lambda}=\kappa_{i}$. Le nombre de telles familles vaut ( $i$ étant fixé)

$$
\prod_{j=1}^{u_{i}}\left(\begin{array}{c}
\kappa_{i, j}+2 d t_{1} a_{i}-1 \\
\kappa_{i, j}-1
\end{array}\right) \leq 2^{\left|\kappa_{i}\right|+2 d t_{1} u_{i} a_{i}} .
$$

TOME $133-2005-\mathrm{N}^{\mathrm{O}} 4$ 
Nous appliquons alors le lemme 5.2 après avoir remarqué que le facteur $\mid x_{j}^{(i)}{ }^{\eta}{ }_{v}$ figurant dans la conclusion va ici disparaître par produit puisque

$$
\prod_{i=1}^{m} \prod_{\lambda=1}^{2 d t_{1} a_{i}}\left|\left(w_{\nu_{\lambda, i}}^{(i)}\right)^{\eta_{\lambda, i}}(x)\right|_{v} \leq 1
$$

en raison du choix de l'indice $j_{v}$ et cela donne (grâce à la formule du produit pour les $c_{i}$ )

$$
\begin{aligned}
& -\phi \leq h(\mathcal{F})+\log \operatorname{dim} E+\sum_{i=1}^{m}\left\{2 d t_{1} u_{i} a_{i} \log 2+\left|\kappa_{i}\right|\left(\log 2 f_{2}\left(u_{i}, D_{i}\right)\right.\right. \\
& \left.\left.+2(N+1) h\left(B_{i}\right)+2(N+1) D_{i} h_{\mathcal{L}}\left(x_{i}\right)\right)\right\} .
\end{aligned}
$$

Par définition de $\kappa$ et notre hypothèse sur $\sigma$, nous avons

$$
\left|\kappa_{i}\right| \leq d t_{1} a_{i} \sigma \leq\left(4(N+1) D_{i}\right)^{-1} d \varepsilon a_{i} .
$$

Nous utilisons aussi l'égalité $\log \operatorname{dim} E=o(d)$ et la majoration $\log 2 f_{2}\left(u_{i}, D_{i}\right) \leq$ $8(N+1) D_{i} \log (N+1) D_{i}$ (calcul immédiat) et donc

$-\phi \leq h(\mathcal{F})+d \sum_{i=1}^{m} a_{i}\left\{2 t_{1} u_{i} \log 2+2 \varepsilon \log (N+1) D_{i}+\frac{\varepsilon}{2} h\left(B_{i}\right)+\frac{\varepsilon}{2} h_{\mathcal{L}}\left(x_{i}\right)\right\}+o(d)$.

Vu la valeur de $\phi$ ceci se réécrit

$$
\begin{aligned}
& \frac{\varepsilon}{2} \sum_{i=1}^{m} a_{i} h_{\mathcal{L}}\left(x_{i}\right)-h_{\mathcal{M}}(x) \\
& \quad \leq \frac{h(\mathcal{F})}{d}+\sum_{i=1}^{m} a_{i}\left\{2 t_{1} u_{i} \log 2+2 \varepsilon \log (N+1) D_{i}+\frac{\varepsilon}{2} h\left(B_{i}\right)\right\}+o(1) .
\end{aligned}
$$

Or, en remarquant $2 t_{1} u_{i} \log 2+2 \varepsilon \log (N+1) D_{i} \leq t_{1} \psi(u) \log \Lambda$, nous pouvons majorer le membre de droite grâce au

Lemme 5.4. - Pour d assez grand, on a

$$
\begin{aligned}
& \frac{h(\mathcal{F})}{d}+\sum_{i=1}^{m} a_{i}\left(h\left(B_{i}\right)+12 t_{1} u \psi(u) \log \Lambda\right) \\
& \quad \leq(8 u \operatorname{dim} X)^{-1} \Lambda^{\omega^{\prime} \psi(u)+m-1-\omega}|a|\left(M t_{2}\right)^{m \operatorname{dim} X-u+1} \max (h(X), \delta) .
\end{aligned}
$$

Démonstration. - Avec la majoration pour $h(\mathcal{F})$, le membre de gauche est au plus

$$
12\left(M t_{2}\right)(N+1)^{m} t_{1} \theta\left(t_{1} m\right)^{u}\left(\prod_{i=1}^{m} D_{i}\right)^{1+\omega} \sum_{i=1}^{m} a_{i}\left(h\left(B_{i}\right)+\delta+4 u \psi(u) \log \Lambda\right)
$$

BULlETIN DE LA SOCIÉtÉ MATHÉMATIQUE DE FRANCE 
pour $d$ assez grand (nous avons utilisé les inégalités $\log \left(2 u_{i}+2\right)<4 u \psi(u) \log \Lambda$ et $\left.4\left(t_{1}+t_{2}+\varepsilon\right)+3 t_{1} \leq 12 t_{1} t_{2}\right)$. D'après le lemme 2.3 , on a

$$
h\left(B_{i}\right) \leq h\left(Y_{i}\right)+D_{i}\left(u_{i}+1\right) \log D_{i}(N+1)
$$

donc

$$
\begin{aligned}
h\left(B_{i}\right)+\delta+4 u \psi(u) \log \Lambda & \leq h\left(Y_{i}\right)+\delta+6 u \psi(u)(\operatorname{deg} X) \Lambda^{\psi(u)-1} \log \Lambda \\
& \leq h\left(Y_{i}\right)+\delta+\frac{2}{3} u(\operatorname{deg} X) \Lambda^{2 \psi(u)-1} \\
& \leq h\left(Y_{i}\right)+\delta+\frac{1}{3} \Lambda^{2 \psi(u)}
\end{aligned}
$$

car $\log \Lambda^{\psi(u)} \leq \frac{1}{9} \Lambda^{\psi(u)}$ (puisque $\Lambda^{\psi(u)} \geq 2^{5}$ ) et $2 u(\operatorname{deg} X) \leq \Lambda$. Ainsi

$$
\sum_{i=1}^{m} a_{i}\left(h\left(B_{i}\right)+\delta+4 u \psi(u) \log \Lambda\right) \leq \Lambda^{2 \psi(u)}\left(M t_{2}\right)^{m \operatorname{dim} X-u}|a| \max (h(X), \delta) .
$$

En majorant $\prod_{i=1}^{m} D_{i} \operatorname{par}(\operatorname{deg} X)^{m} \Lambda^{\psi(u)-1}$, il reste seulement à voir

$$
12(N+1)^{m} t_{1} \theta\left(t_{1} m\right)^{u}(\operatorname{deg} X)^{(1+\omega) m} \leq(8 u \operatorname{deg} X)^{-1} \Lambda^{m}
$$

et cela est clair vu la valeur de $\Lambda$.

Continuant notre calcul, nous avons par application de ce lemme

$$
\frac{1}{2} \varepsilon \sum_{i=1}^{m} a_{i} h_{\mathcal{L}}\left(x_{i}\right)-h_{\mathcal{M}}(x) \leq \frac{1}{4} \Lambda^{\omega^{\prime} \psi(u)+m-1-\omega}|a|\left(M t_{2}\right)^{m \operatorname{dim} X} \max (h(X), \delta) .
$$

D'autre part, il est aisé de vérifier que l'on a $\varepsilon^{-1} \leq \Lambda^{(1+\omega) \psi(u)-\omega}$ ainsi que $(4+2 \omega) \psi(u)+m-1-2 \omega \leq 2 \psi(u-1) \leq 2 \psi(0)$ (par exemple car $m \leq u \psi(u)$ ); par conséquent, le membre de droite de l'inégalité ci-dessus est majoré par $\frac{1}{4} \varepsilon|a| c_{3}$. En utilisant enfin $c_{3} \leq h_{\mathcal{L}}\left(x_{i}\right)$ nous trouvons

$$
\sum_{i=1}^{m} a_{i} h_{\mathcal{L}}\left(x_{i}\right) \leq \frac{4}{\varepsilon} h_{\mathcal{M}}(x)
$$

et cela démontre la proposition 5.3 car la valeur de $c_{1}$ est choisie de sorte que $4 / \varepsilon \leq c_{1}$.

5.3. Théorème du produit. - En vertu de la proposition 5.3, il nous reste, pour conclure la démonstration du théorème 1.2, à montrer que l'on a la majoration $\sigma \leq\left(4 t_{1}(N+1) \max _{i} D_{i}\right)^{-1} \varepsilon$. Nous raisonnons par l'absurde en supposant que cette inégalité est fausse. Le fait que $s$ ait un indice assez grand en $x$ permet alors d'appliquer le théorème du produit et donc de construire un couple $(\ell, U)$ dont l'existence est interdite par la proposition 3.1.

En pratique, l'estimation se fait au but du morphisme $\rho: \mathcal{Y} \rightarrow \mathbb{P}_{\overline{\mathbb{Q}}}^{u_{1}} \times \cdots \times \mathbb{P}_{\overline{\mathbb{Q}}}^{u_{m}}$. Nous commençons donc par déduire de $s$ une section sur ce but.

TOME $133-2005-\mathrm{N}^{\mathrm{O}} 4$ 
LEMME 5.5. - Il existe une section globale $G$ du faisceau

$$
\mathcal{O}\left(d t_{1}\left(\prod_{i=1}^{m} D_{i}\right) a\right)
$$

sur $\mathbb{P}_{\overline{\mathbb{Q}}}^{u_{1}} \times \cdots \times \mathbb{P}_{\overline{\mathbb{Q}}}^{u_{m}}$ d'indice au moins égal à $\sigma$ en $\rho(x)$ relativement aux poids $d t_{1} a$ et telle que

$$
h(G) \leq\left(\prod_{i=1}^{m} D_{i}\right)\left(h(\mathcal{F})+d t_{1} \sum_{i=1}^{m} a_{i}\left(h\left(B_{i}\right)+\log \left(2 u_{i}+2\right)\right)\right)+o(d) .
$$

Démonstration. - D'après les calculs du paragraphe précédent, nous pouvons trouver un couple $(k, \ell)$ tel que $F_{k, \ell}(\Xi)$ est une section de $\mathcal{P}^{\otimes d}$ d'indice $\sigma$ en $x$. Grâce à $j_{1}$, ce polynôme se transforme en un polynôme en $W$ de même hauteur et de multidegré $t_{1} d a$ auquel nous appliquons le lemme 2.5. Par suite, nous avons un polynôme

$$
\sum_{k^{\prime} \in \mathcal{K}} G_{k^{\prime}}(V) W^{k^{\prime}}
$$

où seuls les $V_{j}^{(i)}$ avec $j \leq u_{i}$ interviennent dans $G_{k^{\prime}}$,

$$
h\left(\left(G_{k^{\prime}}\right)_{k^{\prime} \in \mathcal{K}}\right) \leq h(\mathcal{F})+t_{1} d \sum_{i=1}^{m} a_{i}\left(h\left(B_{i}\right)+\log \left(2 u_{i}+2\right)\right)+o(d)
$$

et la section $\alpha=\sum_{k^{\prime}} G_{k^{\prime}}(v) w^{k^{\prime}}$ de $\mathcal{O}_{\mathcal{Y}}$ est d'indice $\sigma$ en $x$.

Considérons la norme de cette section dans l'extension

$$
K(\mathcal{Y}) / K\left(\left(v_{j}^{(i)}\right)_{1 \leq i \leq m, 1 \leq j \leq u_{i}}\right) .
$$

Elle est tout d'abord d'indice au moins $\sigma$ en $x$ car elle s'écrit

$$
N(\alpha)=\prod_{\tau \in \mathcal{T}} \tau(\alpha)=\alpha \prod_{\tau \in \mathcal{T} \backslash\{\text { id }\}} \tau(\alpha)
$$

où $\mathcal{T}$ est l'ensemble des plongements de $K(\mathcal{Y})$ dans une clôture normale de l'extension ci-dessus.

Par ailleurs en développant le produit, il vient

$$
N(\alpha)=\sum_{\left(\lambda_{k^{\prime}}\right)_{k^{\prime} \in \mathcal{K}}}\left(\prod_{k^{\prime} \in \mathcal{K}} G_{k^{\prime}}(v)^{\lambda_{k^{\prime}}}\right) \sum_{\left(\ell_{\tau}\right)_{\tau \in \mathcal{T}}} \prod_{\tau \in \mathcal{T}} \tau(w)^{\ell_{\tau}}
$$

où la première somme porte sur les éléments de $\mathbb{N}^{\mathcal{K}}$ de somme $\operatorname{Card} \mathcal{T}=\prod_{i=1}^{m} D_{i}$ tandis que la seconde concerne les familles de $\mathcal{K}^{\mathcal{T}}$ qui vérifient la condition $\operatorname{Card}\left\{\tau \in \mathcal{T} ; \ell_{\tau}=k^{\prime}\right\}=\lambda_{k^{\prime}}$ pour tout $k^{\prime} \in \mathcal{K}$. En tout état de cause, nous obtenons un polynôme homogène en les $G_{k^{\prime}}$ de degré $\prod_{i=1}^{m} D_{i}$ dont les coefficients sont des polynômes en les $v_{j}^{(i)}\left(1 \leq i \leq m\right.$ et $\left.1 \leq j \leq u_{i}\right)$ indépendants de $d$. 
En conséquence, $N(\alpha)$ s'écrit $G(\alpha)$ où $G$ est multihomogène en ces variables de multidegré $\left(\prod_{i=1}^{m} D_{i}\right) d t_{1} a$ et de hauteur

$$
h(G) \leq\left(\prod_{i=1}^{m} D_{i}\right) h\left(\left(G_{k^{\prime}}\right)_{k^{\prime} \in \mathcal{K}}\right)+o(d) .
$$

Il est possible de voir ce polynôme comme une section de $\mathcal{O}\left(d t_{1}\left(\prod_{i=1}^{m} D_{i}\right) a\right)$ sur $\mathbb{P}_{\overline{\mathbb{Q}}}^{u_{1}} \times \cdots \times \mathbb{P}_{\overline{\mathbb{Q}}}^{u_{m}}$ qui satisfait donc toutes les conditions de l'énoncé.

La forme effective du théorème du produit que nous employons est la suivante :

ThÉORغ̀me 5.6. - Soient $x^{\prime}=\left(x_{1}^{\prime}, \ldots, x_{m}^{\prime}\right)$ un point rationnel du produit $\mathbb{P}_{K}^{u_{1}} \times \cdots \times \mathbb{P}_{K}^{u_{m}}$ et $G$ un polynôme multihomogène de multidegré $\delta^{\prime} \in(\mathbb{N} \backslash\{0\})^{m}$. On suppose que

1) $G$ s'annule en $x^{\prime}$ avec un indice supérieur à $\sigma_{0}$ par rapport $\grave{a}\left(\delta_{1}^{\prime}, \ldots, \delta_{m}^{\prime}\right)$,

2) $\delta_{i}^{\prime} / \delta_{i+1}^{\prime} \geq\left(m / \sigma_{0}\right)^{u}$ et

3) $\sigma_{0} / m \leq\left(2 u^{2}\right)^{-u}$.

Alors il existe un indice $\ell$ et un polynôme non nul $U$ homogène en $V_{0}, \ldots, V_{u_{\ell}}$ tel que

1) $x_{\ell}^{\prime} \in V(U)$

2) $\operatorname{deg} U \leq\left(m / \sigma_{0}\right)^{u}$ et

3) $\delta_{\ell}^{\prime} h(U)$ est majoré par

$$
\begin{gathered}
u_{\ell}\left(\frac{m}{\sigma_{0}}\right)^{u}\left[h(G)+\sum_{i=1}^{m}\left(\delta_{i}^{\prime}\left(h\left(\mathbb{P}_{K}^{u_{i}}\right)+\log 2\right)+\sqrt{u_{i}}\right)+\frac{1}{2}(u-1) \log \left|\delta^{\prime}\right|\right] \\
+\delta_{\ell}^{\prime}\left(\frac{m}{\sigma_{0}}\right)^{u}\left(u_{\ell}+1\right) \log \left(\frac{m}{\sigma_{0}}\right)^{u}\left(u_{\ell}+1\right)+\delta_{\ell}^{\prime} \log \left(\begin{array}{c}
\operatorname{deg} U+u_{\ell} \\
u_{\ell}
\end{array}\right) .
\end{gathered}
$$

Démonstration. - Nous appliquons les résultats de [11]. Il faut remarquer que les énoncés restent tous valables si certains des $u_{i}$ sont nuls. Avec les notations de ce texte, nous avons ici par hypothèse $x^{\prime} \in Z_{\sigma_{0}}(G)$. Nous choisissons une composante irréductible $W$ de $Z_{\sigma_{0}}(G)$ qui contient $x^{\prime}$ et lui appliquons le corollaire 1.2 de [11] (l'hypothèse $\sigma_{0} / m \leq\left(2 u^{2}\right)^{-u}$ assure $\sigma_{0}<m\left(\log (u+1) / 2 u^{2}\right)^{u}$ sauf si $u=1$ mais dans ce cas le corollaire 1.1 montre que $\sigma_{0} \leq m$ suffit). Nous obtenons donc

$$
x^{\prime} \in W \subset Z_{1} \times \cdots \times Z_{m} \varsubsetneqq \mathbb{P}_{K}^{u_{1}} \times \cdots \times \mathbb{P}_{K}^{u_{m}}
$$

où $Z_{i}$ est un sous-schéma fermé intègre de $\mathbb{P}_{K}^{u_{i}}$. Soit $\ell$ un indice pour lequel $Z_{\ell} \neq$ $\mathbb{P}_{K}^{u_{\ell}}$. Nous avons $\operatorname{deg} Z_{\ell} \leq\left(m / \sigma_{0}\right)^{u}$ (voir la remarque à la fin du paragraphe 3 de [11]) et

$\delta_{\ell}^{\prime} h\left(Z_{\ell}\right) \leq u_{\ell}\left(\frac{m}{\sigma_{0}}\right)^{u}\left[h(G)+\sum_{i=1}^{m}\left(\delta_{i}^{\prime}\left(h\left(\mathbb{P}_{K}^{u_{i}}\right)+\log 2\right)+\sqrt{u_{i}}\right)+\frac{1}{2}(u-1) \log \left|\delta^{\prime}\right|\right]$.

TOME $133-2005-\mathrm{N}^{\mathrm{O}} 4$ 
En vertu des lemmes du premier paragraphe de la partie 2, nous pouvons trouver un polynôme $U$ dans l'idéal de $Z_{\ell}$ tel que $\operatorname{deg} U=\operatorname{deg} Z_{\ell}$ et

$$
h(U) \leq h\left(Z_{\ell}\right)+\left(\operatorname{deg} Z_{\ell}\right)\left(u_{\ell}+1\right) \log \left(\operatorname{deg} Z_{\ell}\right)\left(u_{\ell}+1\right)+\log \left(\begin{array}{c}
\operatorname{deg} Z_{\ell}+u_{\ell} \\
u_{\ell}
\end{array}\right) .
$$

Appliqué avec $n$ remplacé par $u_{\ell}$ et $u$ par $\operatorname{dim} Z_{\ell} \leq u_{\ell}-1$, le lemme 2.3 fournit un polynôme de hauteur au plus $h\left(Z_{\ell}\right)+\left(\operatorname{deg} Z_{\ell}\right) u_{\ell} \log \left(\operatorname{deg} Z_{\ell}\right)\left(u_{\ell}+1\right)$ mais dans de nouvelles coordonnées; pour revenir à celles de départ, il faut ajouter la quantité $(\operatorname{deg} U) \log \left(u_{\ell}+1\right) \operatorname{deg} Z_{\ell}+\log \left(\begin{array}{c}\operatorname{deg} U+u_{\ell} \\ u_{\ell}\end{array}\right)$ (voir aussi la démonstration de la proposition 3.1). En multipliant par $\delta_{\ell}^{\prime}$ et en combinant les estimations, le théorème est établi.

Appliquons ce résultat au polynôme $G$ donné par le lemme avec $x^{\prime}=\rho(x)$, $\sigma_{0}=m \Lambda^{-\omega^{\prime} \psi(u)}$ et $\delta^{\prime}=d t_{1}\left(\prod_{i=1}^{m} D_{i}\right) a$. Montrons que les hypothèses sont bien vérifiées. Dans un premier temps, $G$ est d'indice au moins $\sigma$ en $x^{\prime}$ relativement à $d t_{1} a$ donc d'indice au moins $\sigma \prod_{i=1}^{m} D_{i}^{-1}$ relativement à $\delta^{\prime}$. Par la minoration de $\sigma$ (raisonnement par l'absurde) et la valeur de $\varepsilon$, nous avons

$$
\begin{aligned}
\sigma^{-1} \prod_{i=1}^{m} D_{i} & \leq 8 \theta u\left(t_{1} m\right)^{u} t_{1}(N+1) \max _{1 \leq i \leq m} D_{i}\left(\prod_{i=1}^{m} D_{i}\right)^{2+\omega} \\
& \leq 8 \theta u\left(t_{1} m\right)^{u} t_{1}(N+1)(\operatorname{deg} X)^{(2+\omega) m+1} \Lambda^{\omega^{\prime}(\psi(u)-1)} \\
& \leq m^{-1} \Lambda^{\omega^{\prime} \psi(u)}=\sigma_{0}^{-1}
\end{aligned}
$$

Ensuite

$$
\frac{\delta_{i}^{\prime}}{\delta_{i+1}^{\prime}}=\frac{a_{i}}{a_{i+1}} \geq c_{2} \geq \Lambda^{\omega^{\prime} \psi(1)} \geq \Lambda^{\omega^{\prime} u \psi(u)}=\left(\frac{m}{\sigma_{0}}\right)^{u}
$$

car $u \geq 1$ et la fonction $u \mapsto u \psi(u)$ est décroissante. Enfin, la troisième condition est satisfaite car

$$
\frac{\sigma_{0}}{m} \leq \Lambda^{-\omega^{\prime}} \leq\left(2 m^{2} \operatorname{dim}^{2} X\right)^{-m \operatorname{dim} X} \leq\left(2 u^{2}\right)^{-u}
$$

Le théorème s'applique donc et fournit un couple $(\ell, U)$. Pour montrer qu'il remplit les conditions de la proposition 3.1, il reste seulement à majorer $a_{\ell} h(U)$. Or $\delta_{\ell}^{\prime} h(U)$ est facilement plus petit que

$$
u_{\ell}\left(\frac{m}{\sigma_{0}}\right)^{u}\left(h(G)+\sum_{i=1}^{m} \delta_{i}^{\prime}\left(2 u \log \left(m \sigma_{0}^{-1}\right)+\left(u_{i}+1\right) \log \left(u_{i}+1\right)+2\right)\right)+o(d)
$$

BULletin DE LA SOCiÉtÉ MATHÉMATIQUE DE FRANCE 
$\operatorname{car} h\left(\mathbb{P}_{K}^{n}\right) \leq n \log (n+1)$. Grâce à l'estimation pour $h(G)$, il vient en choisissant $d$ assez grand (et en utilisant $u_{i}+1 \leq \Lambda$ et $e^{3} \leq \Lambda$ par exemple)

$$
\begin{aligned}
& a_{\ell} h(U)\left(u_{\ell} \Lambda^{\omega^{\prime} u \psi(u)}\right)^{-1} \\
& \leq \frac{h(\mathcal{F})}{d t_{1}}+\sum_{i=1}^{m} a_{i}\left(h\left(B_{i}\right)+6 u \psi(u) \log \Lambda+\left(u_{i}+1\right) \log \left(u_{i}+1\right)+3\right)+o(d) \\
& \quad \leq \frac{h(\mathcal{F})}{d t_{1}}+\sum_{i=1}^{m} a_{i}\left(h\left(B_{i}\right)+12 u \psi(u) \log \Lambda\right) .
\end{aligned}
$$

Nous pouvons alors employer le lemme 5.4 et cela nous donne exactement l'assertion voulue.

Comme prévu, nous concluons par l'absurde : puisque la proposition 3.1 assure qu'un tel couple $(\ell, U)$ n'existe pas, notre hypothèse sur $\sigma$ est fausse ; par suite, nous avons $\sigma \leq\left(4 t_{1}(N+1) \max _{i} D_{i}\right)^{-1} \varepsilon$ et donc la proposition 5.3 s'applique et nous avons entièrement démontré le théorème 1.2.

\section{BIBLIOGRAPHIE}

[1] BombieRI (E.) - On G-functions, in Recent progress in analytic number theory (Durham 1979), vol. II (Halberstam (H.) \& Hooley (C.), éds.), Academic Press, 1981, pp. 1-67.

[2] The Mordell conjecture revisited, Ann. Scuola Norm. Sup. Pisa Cl. Sci. (4), t. 17 (1990), pp. 615-640, Erratum : ibid. t. 18 (1991), p. 473.

[3] Bost (J.-B.), Gillet (H.) \& Soulé (C.) - Heights of projective varieties and positive Green forms, J. Amer. Math. Soc., t. 7 (1994), pp. 903 1027.

[4] Chardin (M.) - Contributions à l'algèbre commutative effective et à la théorie de l'élimination, Thèse, Université Paris VI, 1990.

[5] Faltings (G.) - Diophantine approximation on abelian varieties, Ann. of Math. (2), t. 133 (1991), pp. 549-576.

[6] The general case of S. Lang's conjecture, in Barsotti Symposium in Algebraic Geometry (Abano Terme, 1991), Perspect. Math., vol. 15, Academic Press, San Diego, CA, 1994, pp. 175-182.

[7] LANG (S.) - Fundamentals of diophantine geometry, Springer-Verlag, 1983.

[8] Poonen (B.) - Mordell-Lang plus Bogomolov, Invent. Math., t. 137 (1999), pp. 413-425.

[9] RÉmond (G.) - Inégalité de Vojta en dimension supérieure, Ann. Scuola Norm. Sup. Pisa Cl. Sci. (4), t. 29 (2000), pp. 101-151.

[10] - Géométrie diophantienne multiprojective, in Introduction to algebraic independence theory (Nesterenko (Y.) \& Philippon (P.), éds.), Lect. Notes in Math., vol. 1752, Springer-Verlag, 2001, pp. 95-131. 
[11] _ Sur le théorème du produit, J. Théor. Nombres Bordeaux, t. 13 (2001), pp. 287-302.

[12] _ Sur les sous-variétés des tores, Compositio Math., t. 134 (2002), pp. 337-366.

[13] _ Approximation diophantienne sur les variétés semi-abéliennes, Ann. Sci. École Norm. Sup. (4), t. 36 (2003), pp. 191-212.

[14] _ Intersection de sous-groupes et de sous-variétés, II, Journal de l'institut de mathématiques de Jussieu, à paraître.

[15] Rémond (G.) \& Viada (E.) - Problème de Mordell-Lang modulo certaines sous-variétés abéliennes, Int. Math. Res. Notices, t. 35 (2003), pp. 1915-1931.

[16] Vojta (P.) - Siegel's theorem in the compact case, Ann. of Math. (2), t. 133 (1991), pp. 509-548.

[17] _ Integral points on subvarieties of semiabelian varieties, I, Invent. Math., t. 126 (1996), pp. 133-181.

[18] Zhang (S.) - Distribution of almost division points, Duke Math. J., t. 103 (2000), pp. 39-46. 